\title{
Glycolytic requirement for NK cell cytotoxicity and cytomegalovirus control
}

\author{
Annelise Y. Mah, ${ }^{1}$ Armin Rashidi, ${ }^{2}$ Molly P. Keppel, ${ }^{1}$ Nermina Saucier, ${ }^{1}$ Emily K. Moore, ${ }^{1}$ \\ Joshua B. Alinger, ${ }^{1}$ Sandeep K. Tripathy, ${ }^{3}$ Sandeep K. Agarwal, ${ }^{4}$ Emily K. Jeng, ${ }^{5}$ Hing C. Wong, ${ }^{5}$ \\ Jeffrey S. Miller, ${ }^{2}$ Todd A. Fehniger, ${ }^{6}$ Emily M. Mace, ${ }^{7}$ Anthony R. French, ${ }^{1}$ and Megan A. Cooper ${ }^{1}$ \\ ${ }^{1}$ Department of Pediatrics, Division of Rheumatology, Washington University School of Medicine, St. Louis, Missouri, USA. \\ 2Department of Medicine, Division of Hematology, Oncology and Transplantation, University of Minnesota, Minneapolis, \\ Minnesota, USA. ${ }^{3}$ Department of Medicine, Division of Gastroenterology, Washington University School of Medicine, \\ St. Louis, Missouri, USA. ${ }^{4}$ Department of Medicine, Division of Immunology, Allergy, and Rheumatology, Baylor College of \\ Medicine, Houston, Texas, USA. ${ }^{5}$ Altor BioScience, Miramar, Florida, USA. ${ }^{6}$ Department of Medicine, Division of Oncology, \\ Washington University School of Medicine, St. Louis, Missouri, USA. Center for Human Immunobiology, Texas Children's \\ Hospital, Baylor College of Medicine, Houston, Texas, USA.
}

NK cell activation has been shown to be metabolically regulated in vitro; however, the role of metabolism during in vivo NK cell responses to infection is unknown. We examined the role of glycolysis in NK cell function during murine cytomegalovirus (MCMV) infection and the ability of IL-15 to prime NK cells during CMV infection. The glucose metabolism inhibitor 2-deoxy-D-glucose (2DG) impaired both mouse and human NK cell cytotoxicity following priming in vitro. Similarly, MCMV-infected mice treated with 2DG had impaired clearance of NK-specific targets in vivo, which was associated with higher viral burden and susceptibility to infection on the C57BL/ 6 background. IL-15 priming is known to alter NK cell metabolism and metabolic requirements for activation. Treatment with the IL-15 superagonist ALT-803 rescued mice from otherwise lethal infection in an NK-dependent manner. Consistent with this, treatment of a patient with ALT-803 for recurrent CMV reactivation after hematopoietic cell transplant was associated with clearance of viremia. These studies demonstrate that NK cell-mediated control of viral infection requires glucose metabolism and that IL-15 treatment in vivo can reduce this requirement and may be effective as an antiviral therapy.

Conflict of interest: HCW and EKJ are employees and stockholders of Altor BioScience Corporation. JSM consults for Celgene, Fate Therapeutics, and Oxis Biotech. TAF consults for CytoSen Therapeutics and has research funding from Affimed, Celgene, and Altor Bioscience.

Submitted: May 30, 2017

Accepted: October 26, 2017

Published: December 7, 2017

Reference information: JCI Insight. 2017;2(23):e95128. https://doi.org/10.1172/jci. insight. 95128 .

\section{Introduction}

It is now recognized that metabolism plays a key role in the activation and specialized functions of immune cells. Cells fuel ATP production through 2 major metabolic pathways: glycolysis (the metabolic breakdown of glucose) and oxidative phosphorylation (the synthesis of ATP in the presence of oxygen). For T cells, macrophages, and DCs, inflammatory activation is associated with metabolic reprogramming from a relatively metabolically quiescent state to a highly glycolytic state (1-4). These metabolic changes are thought to assist the generation of biosynthetic precursors for effector functions. For example, high glycolytic flux can produce nucleotide precursors for cell division in T cells (5), citrate for production of ROS in macrophages (6), and lipid precursors for ER membrane synthesis in DCs (7). Glycolytic enzymes have also been shown to directly regulate cytokine production and gene expression in T cells $(8,9)$. Thus, in many immune cell types, metabolic regulation equips activated cells to carry out specialized functions.

NK cells are innate cytotoxic lymphocytes that are first responders against virally infected cells and tumor cells. They respond within hours of stimulation by releasing cytokines, such as IFN- $\gamma$, and killing target cells. Stimulation with cytokines for longer periods, including inflammatory cytokines such as IL-12 and IL-15, significantly enhances NK cell functions. Recent studies have demonstrated that certain NK cell responses require metabolic cues. Naive NK cells rely on glucose-fueled oxidative phosphorylation, while expansion with IL-15 elevates metabolic rates with a preference toward glycolysis, similar to changes seen during the activation of $\mathrm{CD}^{+} \mathrm{T}$ cells $(10,11)$. Several studies show that NK cell production of the cytokine IFN- $\gamma$ is regulated by metabolism $(10,12-14)$. We previously demonstrated that, in freshly isolated murine NK cells, IFN- $\gamma$ production after short-term receptor activation is highly dependent on glycolytic 
and oxidative metabolism, while IFN- $\gamma$ production stimulated by cytokine activation (IL-12 + IL-18) is independent of both metabolic pathways. We found that receptor activation depends less on these metabolic pathways after priming with high-dose IL-15 (10). Others have found that after expansion in IL-15 and stimulation with IL-2 + IL-12, highly activated lymphokine-activated killer (LAK) cells require glycolysis for maximal production of IFN- $\gamma$ and the cytotoxic granule protein granzyme B (Gzmb) (12). Studies in human NK cells show a similar pattern - IFN- $\gamma$ and Gzmb production downstream of certain stimuli is sensitive to metabolic inhibition (13). Together, these studies demonstrate that NK cells have specific metabolic requirements for IFN- $\gamma$ and Gzmb production. Few studies have directly examined the metabolic regulation of other NK cell functions, such as target killing and proliferation. Here, we investigate the role of glycolysis in NK cell cytotoxicity in vitro and in vivo in response to viral infection.

NK cells are particularly important for antiviral responses against cytomegalovirus. In response to human cytomegalovirus (HCMV), NK cells bearing the activating NKG2C receptor rapidly expand and persist $(15,16)$. These cells, termed CMV-adapted NK cells, display enhanced responses, particularly when triggered by antibodies through their Fc receptor (17-20). While the adaptive T cell response to HCMV is required for long-term immunity, evidence from $\mathrm{B}$ cell- and $\mathrm{T}$ cell-deficient patients demonstrates that the expansion of $\mathrm{NKG}_{2} \mathrm{C}^{+} \mathrm{NK}$ cells alone can control HCMV infection (21).

Acute infection with murine cytomegalovirus (MCMV) in C57BL/6 mice is well described to be controlled by NK cells (22-26). During the first 2 days of infection, NK cells are activated by circulating cytokines and respond by producing a burst of immunomodulatory cytokines, most notably IFN- $\gamma$ (27). After day 2 , expression of the virally encoded ligand $\mathrm{m} 157$ on the surface of infected cells leads to the expansion of $\mathrm{Ly} 49 \mathrm{H}^{+} \mathrm{NK}$ cells, which specifically recognize and kill m157-expressing cells (28-31). Thus, this murine system can be used to examine NK cell activation and proliferation, and the killing of infected cells in vivo; it is also a model for human infection.

In the current study, we found that NK cell proliferation and cytotoxicity following IL-15 priming in vitro were impaired when NK cells were cultured with the glucose metabolism inhibitor 2-deoxy-D-glucose (2DG), which blocks the first 2 enzymes of glycolysis. Glycolytic inhibition decreased expression of cytotoxic proteins and altered the adhesion of NK cells to target conjugates. In vivo, treatment of MCMV-resistant C57BL/6 mice with 2DG caused significant susceptibility to infection and was associated with impaired NK-mediated clearance of $\mathrm{m} 157^{+}$targets. Similar susceptibility to infection was also observed when animals were treated with rapamycin, a drug that inhibits the mTOR pathway. Priming with the IL-15 superagonist ALT-803 rescued 2DG- or rapamycin-treated animals from lethal infection. 2DG also decreased human NK cell cytotoxicity in vitro; here, we describe a hematopoietic cell transplant (HCT) patient with recurrent CMV reactivation treated with ALT-803. Similar to findings in our murine model, ALT-803 treatment in this patient was associated with clearance of viremia, suggesting therapeutic potential for IL-15 agonists in CMV.

\section{Results}

Inhibiting glucose metabolism with 2DG decreases NK cell proliferation and cytotoxicity in vitro. Murine NK cells require activation, such as priming with IL-15, to upregulate cytotoxic effector molecules, such as Gzmb, and effectively kill target cells (32). To investigate the requirement for glucose metabolism in NK cytotoxicity, NK cells were purified from WT C57BL/6 mice and plated in $100 \mathrm{ng} / \mathrm{ml} \mathrm{IL-15}$ with or without 1 $\mathrm{mM} 2 \mathrm{DG}$ for 72 hours. This dose of 2DG maintained NK cell viability, with minimal differences between treated and untreated cells (Supplemental Figure 1A; supplemental material available online with this article; https://doi.org/10.1172/jci.insight.95128DS1). Extracellular flux assays confirmed that 2DG treatment significantly decreased NK cell glycolysis, as measured by the extracellular acidification rate (ECAR) (Figure 1A). We previously demonstrated that naive murine NK cells primarily use glucose-based oxidative phosphorylation (10). Consistent with this, 2DG treatment also decreased the oxygen consumption rate (OCR) (Figure 1A). 2DG significantly inhibited IL-15-induced proliferation (Figure 1B), leading to a reduction in the number of cells recovered at the end of the incubation (Supplemental Figure 1B).

The ability of NK cells to kill target cells after priming with IL-15 with or without 2DG was measured at various effector-to-target ratios (E:T ratios) in complete media. Ba/F3-m157 cells are murine pro-B cells ectopically expressing the MCMV-encoded protein $\mathrm{m} 157$ that is recognized by $\mathrm{Ly} 49 \mathrm{H}^{+} \mathrm{NK}$ cells, and killing of Ba/F3-m157 is mediated solely by Ly49H-expressing NK cells $(29,33,34)$. Killing of Ba/F3-m157 targets was significantly impaired when activated in the presence of 2DG (Figure 1C). NK cell killing of 

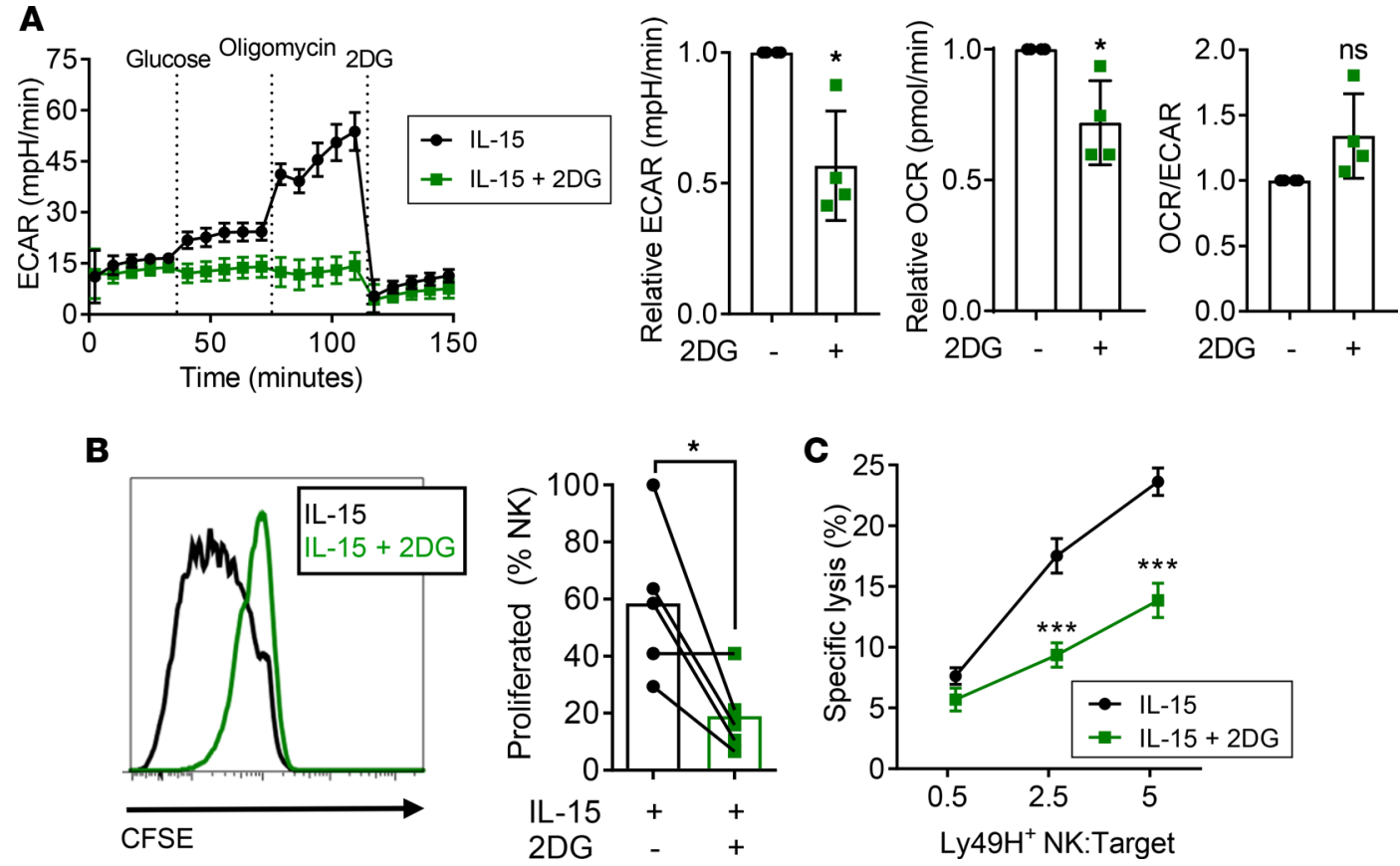

Figure 1. Glucose metabolism blockade during activation decreases NK cell proliferation and cytotoxicity. Purified NK cells were cultured for 72 hours in $100 \mathrm{ng} / \mathrm{ml} \mathrm{IL-15}$ without (black) or with $1 \mathrm{mM}$ 2-deoxy-D-glucose (2DG, green). (A) Representative extracellular flux assay showing glycolytic rate as estimated by extracellular acidification rate (ECAR); glycolytic stress test shows baseline ECAR in glucose-free media and after addition of glucose, maximal ECAR after addition of oligomycin, and nonglycolytic acidification after 2DC. Summary data shows ECAR, oxygen consumption rate (OCR), and the OCR/ ECAR ratio normalized to control ( $n=4$ /group in 3 separate experiments, ratio paired $t$ test performed on raw data). (B) Representative histogram shows CFSE dilution of proliferating NK cells. Summary graph shows the percentage of NK cells proliferated ( $n=5$ experiments, paired $t$ test). (C) NK cells activated in IL-15 $\pm 2 \mathrm{DC}$ were assessed for their ability to kill Ba/F3-m157 target cells at different effector/target ratios (ratios represent Ly49H $\mathrm{NK}^{+}$cells/targets), $n=8$ /group, 4 experiments, 2 -way ANOVA. Data show mean \pm SEM. ${ }^{*} P<0.05,{ }^{* *} P<0.001$.

MHC class I-deficient RMA-S tumor cells $(35,36)$ was also significantly impaired by 2 DG. Interestingly, killing of YAC-1 tumor cells, which are primarily recognized by NKG2D (37-39), was not impaired by 2DG (Supplemental Figure 1C). These results suggest that glycolytic inhibition caused target-specific deficits in NK cell killing, possibly based on different mechanisms of target cell recognition and NK activation.

Decreased killing by $2 D G$-treated $N K$ cells is associated with low Gzmb and variable actin accumulation during target conjugation. We investigated several mechanisms by which 2DG might decrease NK cell cytotoxicity. First, IL-15 increases levels of cytotoxic granule proteins in NK cells (32). Treatment with 2DG reduced expression of Gzmb (Figure 2A), suggesting one potential mechanism for decreased NK cell cytotoxicity. Next, we investigated the dynamics of NK cell recognition and killing of target cells. Target cell killing is a tightly orchestrated event composed of multiple steps including recognition of the target cell, adhesion, and formation of an immunological synapse (IS), anchoring to the target with filamentous F-actin, convergence of granules to the microtubule organizing center (MTOC), polarization and trafficking of the MTOC toward the IS, degranulation, and finally detachment (40). By flow cytometry, 2DG did not significantly decrease NK cell recognition of potential targets, as demonstrated by normal conjugate formation between 2DG-treated NK cells and Ba/F3-m157 targets (Figure 2B). Confocal microscopy of cocultured NK cells and $\mathrm{Ba} / \mathrm{F} 3-\mathrm{m} 157$ targets also demonstrated conjugate formation by 2DG-treated NK cells (Figure 2C). In some conjugates in both treatment groups, Ly49H was detected at the synapse (Figure 2D). However, actin accumulation at the IS was highly variable among 2DG-treated NK cells, indicating that 2DG may impair proper adhesion to the target (Figure 2, C and E). Compared with control-treated NK cells, most 2DG-treated NK cells had negative actin accumulation, indicating abnormal anchoring to the target and impaired commitment to killing (Figure 2E). However, 2DG did not affect polarization of the MTOC, as measured by the distance of the MTOC to the IS (Figure 2F), suggesting that once NK cells committed to killing, MTOC polarization was not impaired. Thus, these data demonstrate that inhibition of glucose metabolism interfered with NK cell priming, leading to decreased levels of Gzmb protein production and altered actin accumulation at the IS. 
A

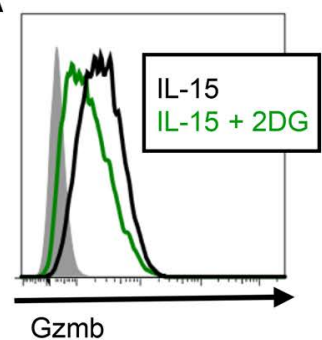

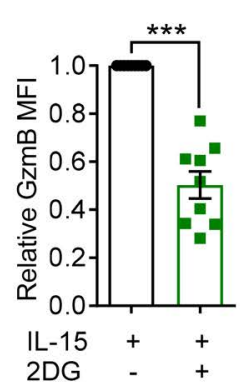

B

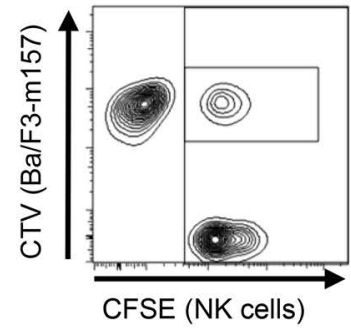

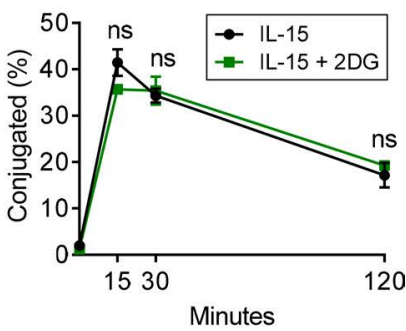

D
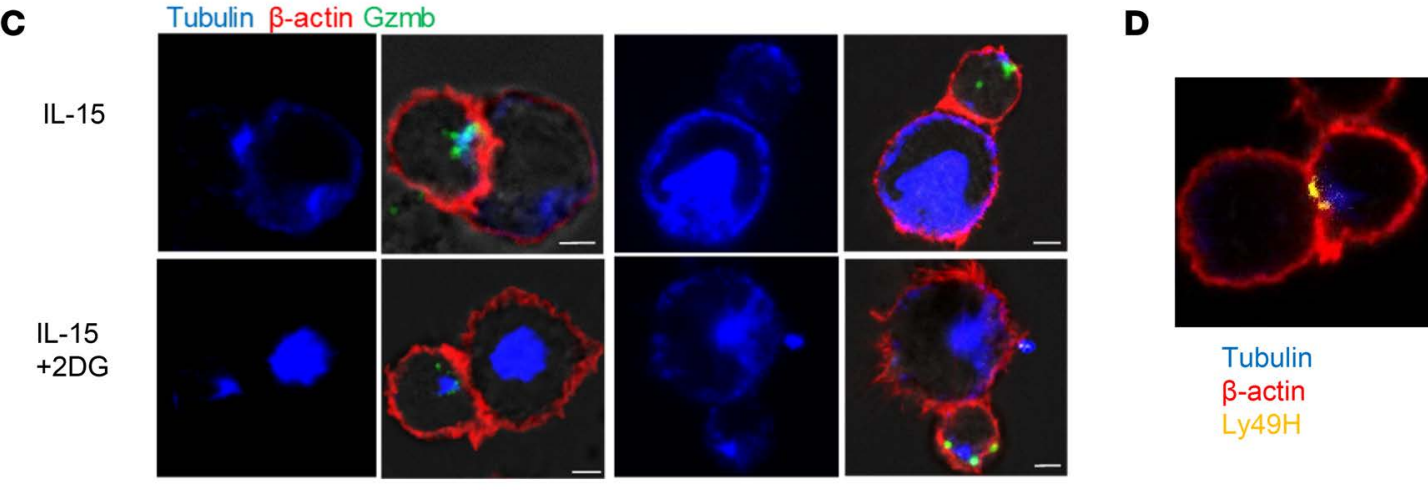

Tubulin

$\beta$-actin Ly $49 \mathrm{H}$

E

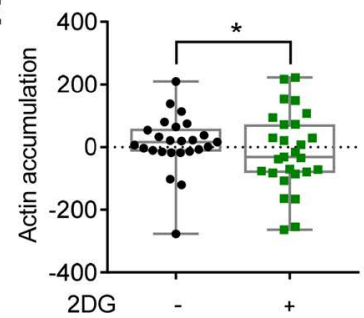

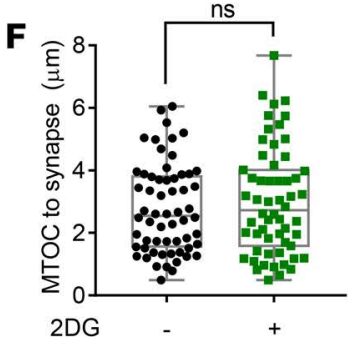

Figure 2. In vitro treatment with 2DC decreases NK cell production of granzyme B and alters actin accumulation at the immunological synapse. Purified NK cells were cultured for 72 hours in $100 \mathrm{ng} / \mathrm{ml} \mathrm{IL-15}$ with or without $1 \mathrm{mM} 2$-deoxy-D-glucose (2DC). (A) Representative flow histogram shows granzyme B (Gzmb) levels in naive (filled) and activated NK cells cultured without (black) or with (green) $1 \mathrm{mM} 2 \mathrm{DG}$. Summary data are normalized to mean fluorescence intensity (MFI) of naive NK cells ( $n=9 /$ group, 5 separate experiments, ratio paired $t$ test performed on raw data). (B) NK cells were incubated at a 1:1 ratio with $\mathrm{Ba} / \mathrm{F3}-\mathrm{m} 157$ target cells for various times; conjugates were detected by flow cytometry. Representative flow cytometry shows CFSE ${ }^{+}$and CellTrace Violet (CTV) ${ }^{+}$conjugates, and conjugation curve shows percentage of CFSE ${ }^{+}$cells conjugated $(n=2-3$ technical replicates, representative of 3 separate experiments, 2-way ANOVA). (C-F) NK cells were incubated with Ba/F3-m157 cells for 40 minutes at a 2:1 ratio. (C and D) Fluorescence microscopy of NK:Ba/ F3-m157 conjugates show convergence of granules (green, Gzmb), Ly49H accumulation at the synapse (yellow), polarization of the microtubule organizing center (MTOC, blue) toward the synapse, and actin accumulation (red). Scale bar: $2 \mu \mathrm{m}$. Representative images for the range of control and 2DG-treated NK cells are shown. (E) Quantification of actin accumulation by area $x$ intensity of synapse - (NK + target) ( $n=25-27$ conjugates/group, 4 experiments, distribution analyzed by Kolmogorov-Smirnov test). (F) Quantification of MTOC-synapse distance in $\mu \mathrm{m}$, not significant by 2-tailed Welch's $t$ test for means or Kolmogorov-Smirnov test ( $n=58-60$ conjugates/group, 5 experiments). For $\mathbf{A}$ and $\mathbf{B}$, data show mean \pm SEM. For $\mathbf{E}$ and $\mathbf{F}$, the gray box displays median, 25th, and 75th percentiles. ${ }^{*} P<0.05,{ }^{* * *} P<0.001$.

2DG-treated, MCMV-infected mice have impaired target clearance day 2 after infection. To examine the effects of glycolytic inhibition on NK cell function in vivo, we treated MCMV-infected mice with 2DG. Female WT mice were infected i.p. with $1 \times 10^{5} \mathrm{PFU}$ of $\mathrm{MCMV}\left(\sim 40 \% \mathrm{LD}_{50}\right)(41)$ and injected daily with either $19 \mathrm{mg}(1 \mathrm{~g} / \mathrm{kg})$ of $2 \mathrm{DG}$ or vehicle (PBS). To determine whether 2DG administration altered NK cell cytotoxicity, in vivo target clearance was assessed. Syngeneic WT splenocytes (nontargets) and transgenic splenocytes (targets) expressing the MCMV-encoded activating ligand $\mathrm{m} 157$, which is only recognized by Ly $49 \mathrm{H}^{+} \mathrm{NK}$ cells, were differentially labeled and adoptively transferred into infected mice 2 days after infection (42). Clearance of targets in 2DG-treated MCMV-infected mice was significantly lower than in PBS-treated MCMV-infected mice and was similar to baseline clearance in mock-infected mice (Figure $3 \mathrm{~A})$. This finding suggests that 2DG treatment impaired NK cell activation with MCMV infection and subsequent killing of target cells. 


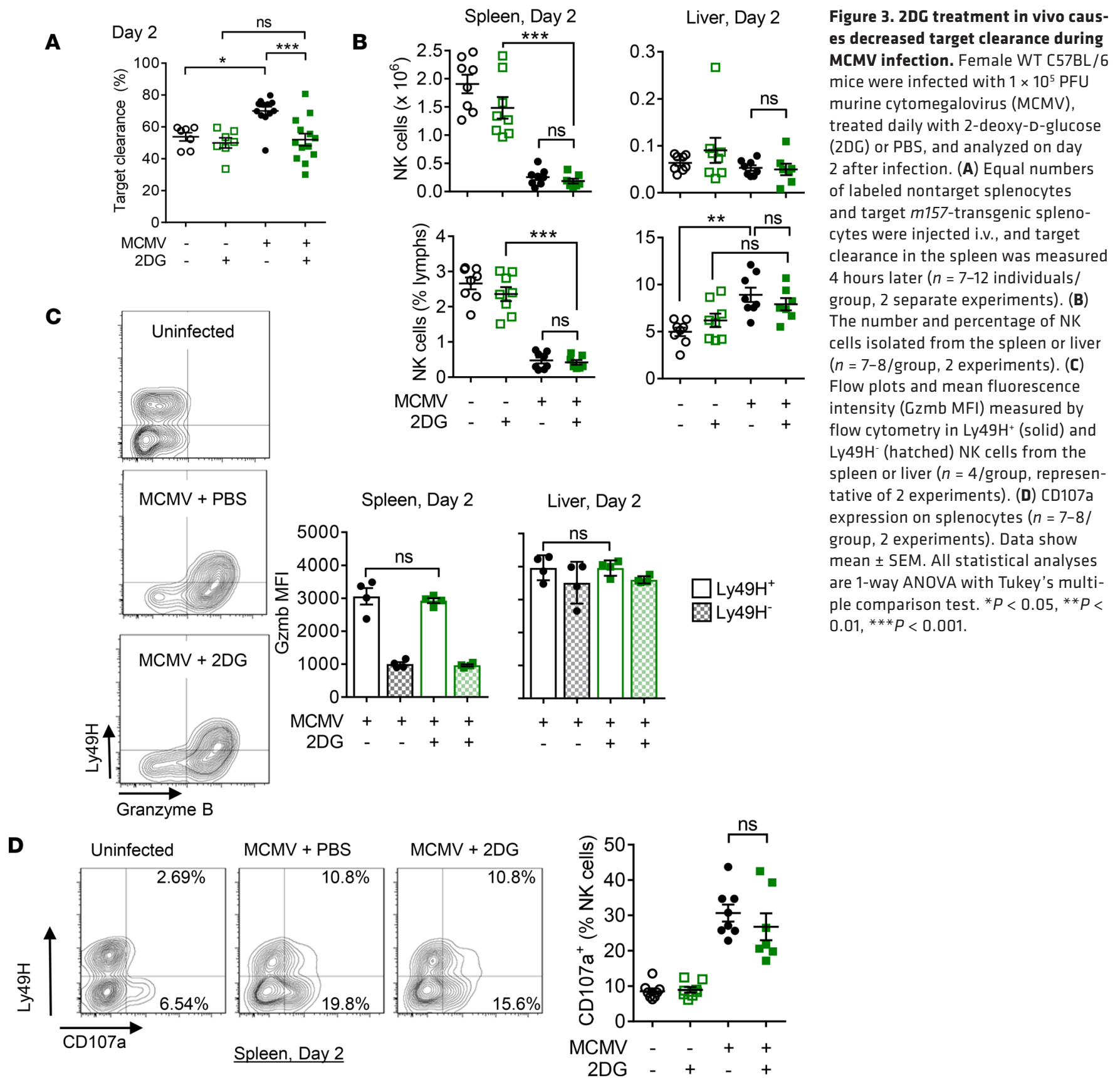

The defect in target clearance in 2DG-treated mice was not due to differences in NK cell numbers, as there were no significant differences in the number or percentage of NK cells (Figure 3B), or in Ly49H expression on NK cells (not shown), in the spleens or livers at this early time point. To determine whether NK cells had a defect in cytotoxic capacity, intracellular Gzmb and degranulation, as measured by cellsurface expression of CD107a, were tested. NK cells rapidly upregulate Gzmb and other cytotoxic proteins after infection with MCMV, and levels of Gzmb at day 2 in NK cells from infected mice were significantly higher than in naive mice. However, there were no differences between 2DG- and PBS-treated mice in the percentage of $\mathrm{Gzmb}^{+} \mathrm{NK}$ cells or in the amount of $\mathrm{Gzmb}$ protein as measured by mean fluorescence intensity (MFI) (Figure 3C). NK degranulation measured by cell-surface level of CD107a stained directly ex vivo was equivalent between 2DG-treated and PBS control mice (Figure 3D). Similarly, no differences in T cell numbers, Gzmb expression, or degranulation were detected (Supplemental Figure 2, A-C). These results suggest that NK cells from 2DG-treated mice retained cytotoxic machinery but were unable to mount productive target cell killing. 
A

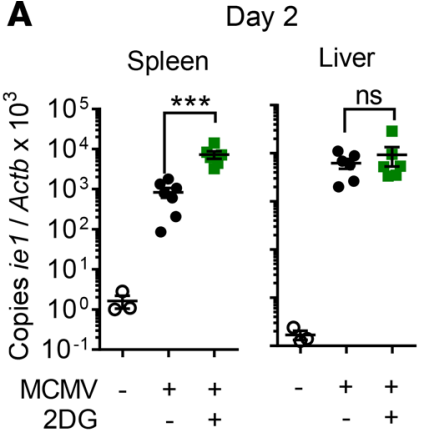

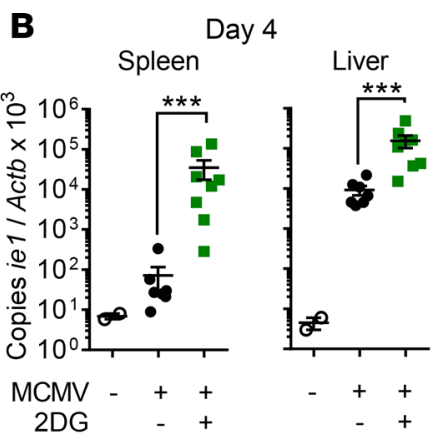

Spleen, Day 4

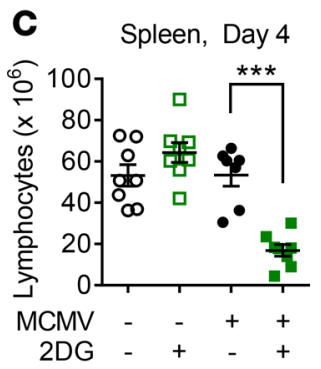

Liver, Day 4
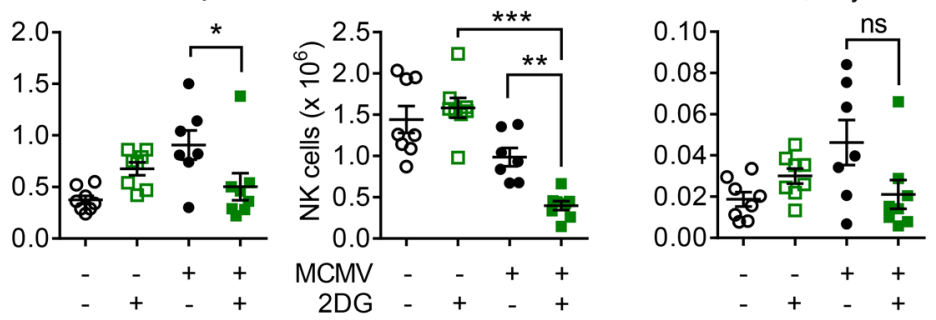

D
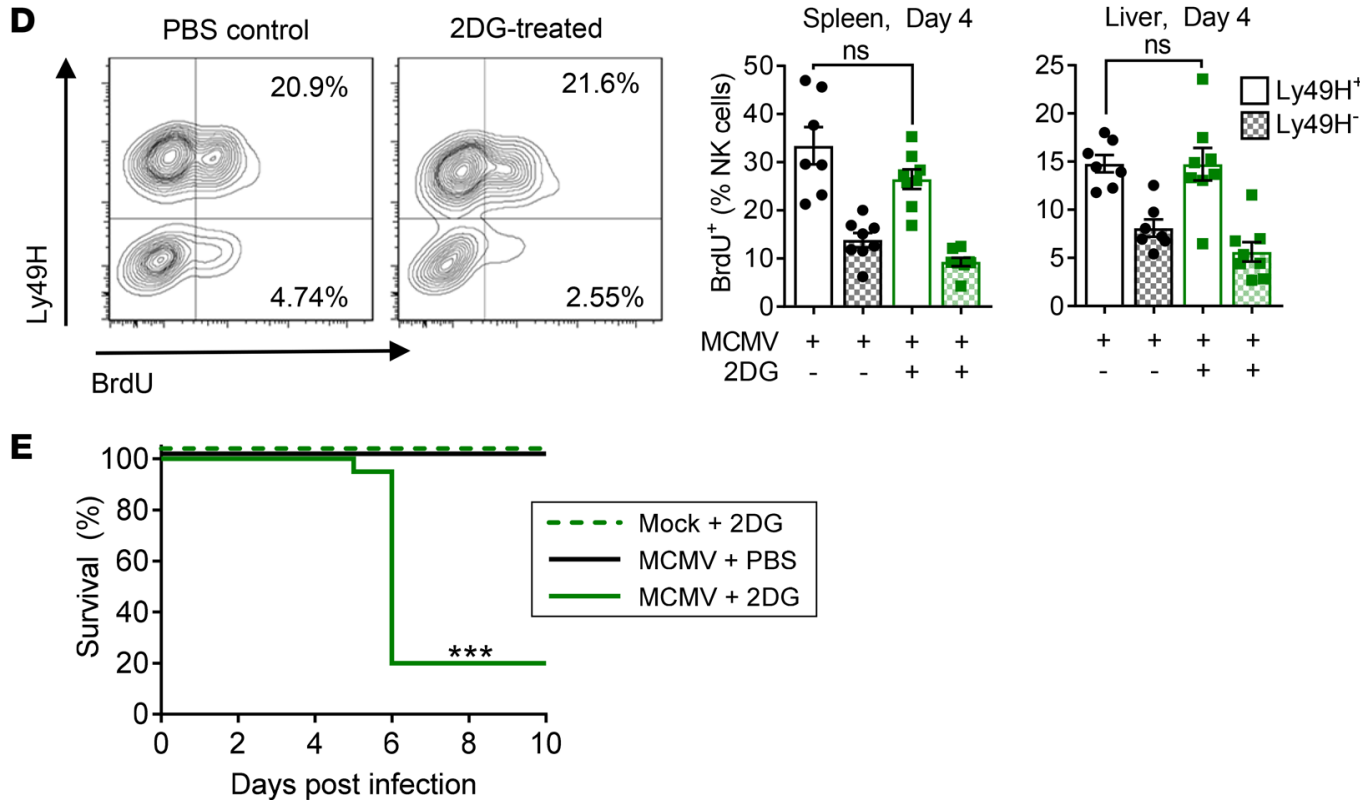

Figure 4. 2DG treatment in vivo confers MCMV susceptibility. Female WT C57BL/ 6 mice were infected with $1 \times 10^{5}$ PFU murine cytomegalovirus (MCMV) and treated daily with 2-deoxy-D-glucose (2DC) or PBS. Viral copy number by quantitative PCR from the spleen and liver was measured on (A) day 2 or (B) day 4 after infection, shown as copies of MCMV ie1 gene/copies of $\beta$-actin $\times 1,000$ ( $n=3-8$ individuals/group, 2 experiments, 1-way ANOVA on log-transformed data). (C) Total numbers of lymphocytes and NK cells from the spleen or liver at day 4 after infection. (D) Representative flow cytometry and summary data showing the percentage of BrdU ${ }^{+} \mathrm{Ly}_{4} 9 \mathrm{H}^{+} \mathrm{NK}$ cells (open) and $\mathrm{Ly}_{49 \mathrm{H}^{-}} \mathrm{NK}$ cells (hatched), assessed 3 hours after BrdU injection. For $\mathbf{C}$ and $\mathbf{D}$, $n=7-8$ /group, 2 experiments, 1-way ANOVA with Tukey's multiple comparison test. (E) Survival of uninfected mice compared with infected mice treated with 2DG or PBS ( $n=20$ /group, 4 experiments, log-rank Mantel-Cox test). Data shown as mean \pm SEM or survival. ${ }^{*} P<0.05,{ }^{* *} P<0.001$.

NK cells in 2DG-treated mice produce normal levels of IFN- $\gamma$ during MCMV infection. NK cell production of IFN- $\gamma$ peaks at 1.5 days after MCMV infection in response to systemic release of inflammatory cytokines, including IL-12 made by DCs (43). While others have observed that IFN- $\gamma$ production by LAK cells in vitro or by poly(I:C)-stimulated NK cells in vivo is susceptible to inhibition by 2DG (12), we previously demonstrated in vitro that naive NK cell production of cytokine-driven IFN- $\gamma$ is not inhibited by 2DG or by culture in glucose-free media (10). In agreement with our previous in vitro studies, there was no difference in IFN- $\gamma$ production during MCMV infection with 2DG treatment (Supplemental 
Figure 3). These data also suggest an intact innate immune cytokine response to MCMV in the context of systemic 2DG injection, since early NK cell IFN- $\gamma$ production is dependent on cytokines produced by antigen-presenting cells.

$2 D G$ treatment causes susceptibility to MCMV infection. To test whether impaired NK cell clearance of targets correlates with susceptibility to infection, MCMV copy number was assessed in the spleen and liver. 2DG treatment was associated with higher viral burdens as early as day 2 after infection in the spleen (Figure 4A), and by day 4, there was significant loss of viral control, evidenced by high viral copy number in both the spleen and liver (Figure 4B). Data on organ-specific control of MCMV is mixed, but one hypothesis is that NK cell IFN- $\gamma$ is more important for control of MCMV in the liver, while NK cell cytotoxicity and $\mathrm{Ly} 49 \mathrm{H}$ are responsible for control of infection in the spleen (44-47), which is consistent with our findings.

By day 4 after infection, 2DG treatment and high viral burden led to a decrease in the total number of splenocytes and liver lymphocytes (Figure 4C), with correspondingly decreased numbers of NK cells. There were no differences in the numbers of lymphocytes or NK cells with 2DG treatment alone, and cell loss was specifically associated with uncontrolled MCMV infection in 2DG-treated mice. Low NK cell numbers at this time point were not due to decreased NK cell proliferation, since the percentage of NK cells with BrdU incorporation at day 4 after infection was similar with and without 2DG treatment (Figure 4D). T cell numbers and BrdU incorporation were also similar with 2DG treatment (Supplemental Figure 2, D and E). Thus, 2DG did not affect in vivo receptor-mediated proliferation of $\mathrm{Ly} 49 \mathrm{H}^{+} \mathrm{NK}$ cells, but it did inhibit in vitro IL-15-stimulated proliferation.

Loss of viral control with 2DG treatment was associated with significant mortality, with $80 \%$ of normally resistant female B6 mice dying between days 5 and 6 after infection (Figure 4E). 2DG treatment alone without infection had no effect on survival. Mortality was dependent on both the dosage of virus and drug, as lower doses of MCMV or 2DG did not lead to mortality (Supplemental Figure 4). Together, these data show that glucose metabolism was crucial in the clearance of an NK-dependent viral infection.

IL-15 can rescue MCMV-infected, 2DG-treated mice. In vitro, culture with IL-15 enhances NK cell metabolism, preferentially glycolysis, and confers some resistance to the effects of metabolic inhibition; specifically, IFN- $\gamma$ production in glucose-free media was partially restored if NK cells were primed with IL-15 (10). Since NK cell cytotoxicity was susceptible to glycolytic inhibition, we tested whether IL-15 could mitigate NK cell dependence on glucose during MCMV infection. Mice were treated with the human IL-15 superagonist complex ALT-803, which is composed of IL-15 with the N72D mutation and IL-15R $\alpha$ fused to the Fc of IgG1. ALT-803 has been shown to activate NK cells and CD8 ${ }^{+} \mathrm{T}$ cells, and it exhibited antitumor and antiretroviral activities in various experimental animal models (48-57). Mice were treated with $5 \mu \mathrm{g}$ of ALT-803 at 3 and 1 days prior to infection, which increases the number and percentage of NK cells in the spleen by 2 - to 10 -fold on the day of infection (data not shown). ALT-803 treatment significantly increased the baseline and maximal glycolytic and oxidative capacity of NK cells, but it did not alter the ratio of oxidative phosphorylation to glycolysis (OCR/ECAR), suggesting that ALT-803-activated NK cells had high metabolic potential and activity but did not especially favor glycolysis (Figure 5A). This is in contrast to IL-15 treatment in vitro, which we previously demonstrated decreased the OCR/ECAR ratio (10).

Treatment with ALT-803 rescued the deficit in NK cell target clearance at day 2 after infection in MCMV-infected, 2DG-treated mice (Figure 5B). ALT-803 treatment increased the total number of splenic NK cells and Gzmb expression, but neither of these was affected by 2DG (Figure 5, C and D). There was no difference in splenic MCMV copy number at day 2 after infection between 2DG-treated and PBS control mice that had been pretreated with ALT-803 (Figure 5E), suggesting enhanced viral control as compared with 2DG treatment alone (Figure 4A). However, liver MCMV copy number was increased with 2DG and ALT-803 treatment as compared with ALT-803 alone (Figure 5E). The difference in liver MCMV burden appears to be due to decreased MCMV copy numbers in the liver of ALT-803-treated mice, when compared with those with MCMV infection alone (Figure 4A), and due to the loss of this protective effect with 2DG treatment". To assess survival, mice were monitored and received an additional dose of ALT-803 at day 2 after infection. Mice receiving ALT-803 were completely protected from MCMV infection with 2DG treatment (Figure 5F). Thus, treatment with ALT- 803 rescued both the target clearance and survival deficits induced by 2DG.

IL-15 rescue is dependent on $N K$ cells. To determine whether enhanced target clearance and survival with ALT-803 were NK-dependent, NK cells or CD8 ${ }^{+} \mathrm{T}$ cells - the 2 major cell types responsive to IL-15 - were antibody depleted ( $\alpha-\mathrm{NK} 1.1$ or $\alpha-\mathrm{CD} 8 \mathrm{~b})$. Mice depleted of NK or CD8 ${ }^{+}$cells, or control-treated 
A
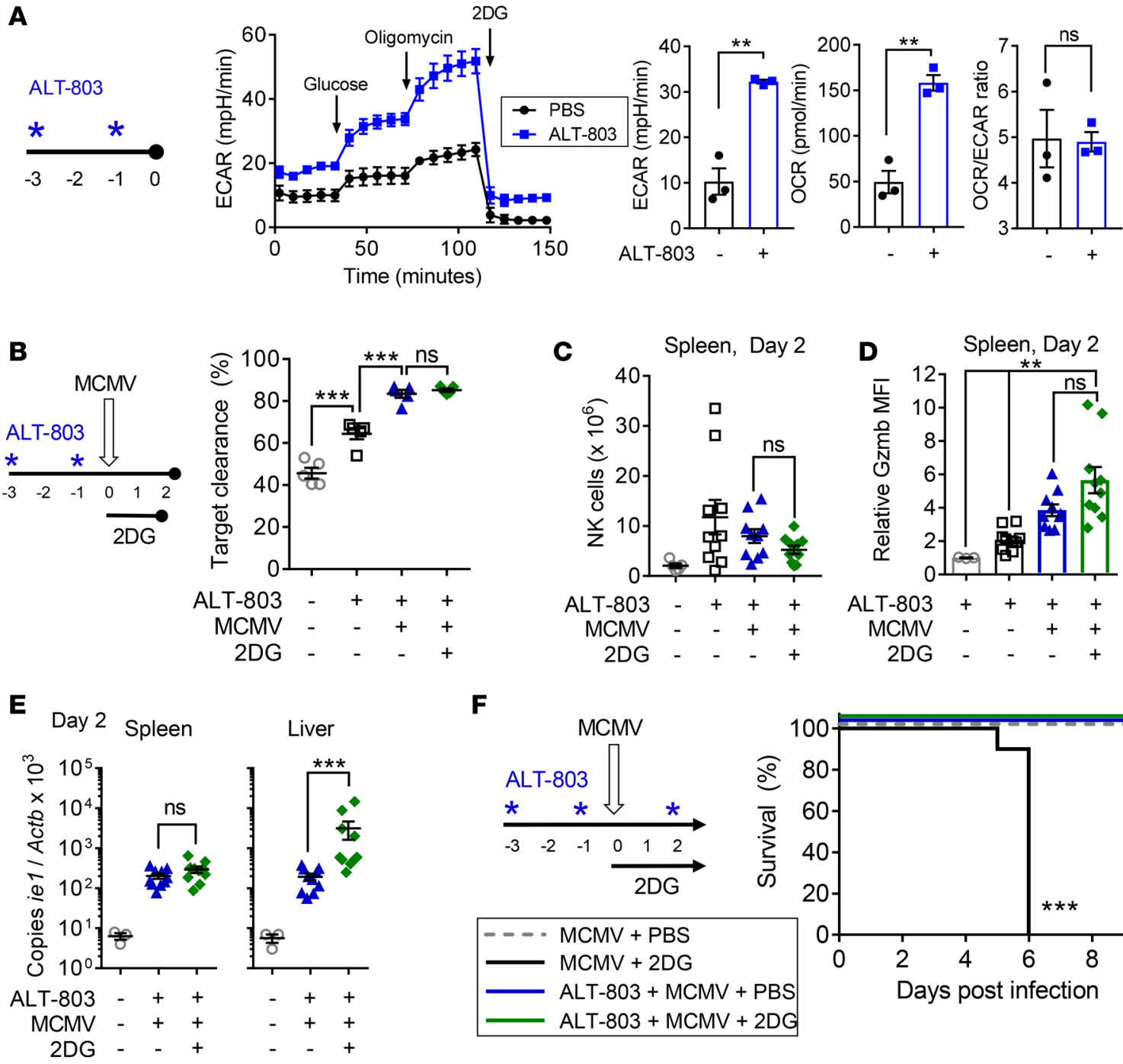

$\mathbf{F}$
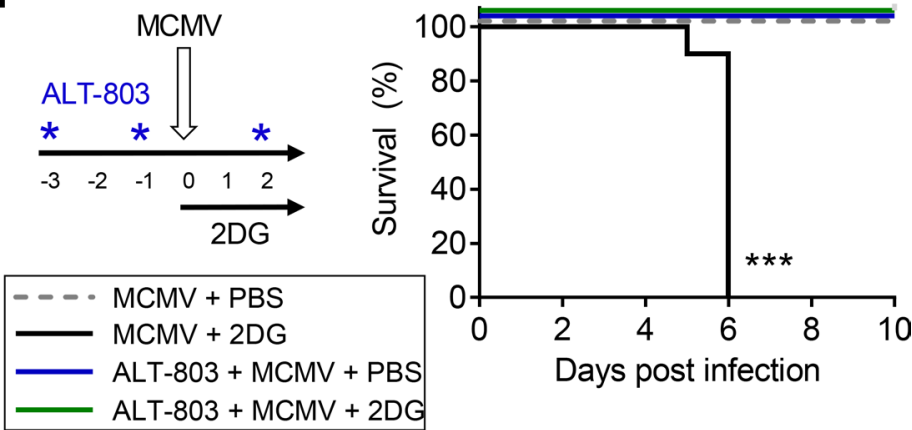

Figure 5. NK activation with ALT-803 rescues MCMV susceptibility caused by 2DC treatment. (A) Female WT C57BL/6 mice were treated with $5 \mu \mathrm{g}$ ALT-803 twice, NK cells were purified from their spleens, and a glycolytic stress test was performed. Representative graph showing baseline, normal (+glucose), and maximal (+oligomycin) extracellular acidification rate (ECAR) in control vs. ALT-803-treated mice. Average ECAR, oxygen consumption rate (OCR), and OCR/ECAR ratio are shown after glucose addition ( $n=3$ individuals/group, 2 separate experiments, 2-tailed $t$ test). (B-E) ALT-803-treated mice were infected with $1 \times 10^{5} \mathrm{PFU}$ murine cytomegalovirus (MCMV) and treated with 2-deoxy-D-glucose (2DC) daily. (B) Clearance of m157-transgenic targets from the spleens of infected and uninfected mice \pm ALT- 803 and $2 D C$ on day 2 after infection ( $n=5$ /group, representative of 2 experiments, 1-way ANOVA). (C) The number and percentage of NK cells isolated from the spleens or livers of mice in B. (D) Mean fluorescence intensity of granzyme B (Gzmb MFI) in all NK cells from B. (E) Viral copy number measured by quantitative PCR from the spleens and livers collected in B, shown as copies of MCMV ie1 gene/copies of $\beta$-actin $\times 1,000$. For $\mathbf{C}-\mathbf{E}, n=10$ /group, 2 experiments, 1-way ANOVA, using log-transformed data for $\mathbf{E}$. (F) Mice were given an additional dose of ALT-803 2 days after infection and followed for 10 days. Survival of mice with no treatment (gray dashed line), 2DG treatment (black), ALT-803 treatment (blue), or ALT-803 with 2DG (green) ( $n=10$ /group, 2 experiments, log-rank Mantel-Cox test). Data shown as mean $\pm \mathrm{SEM}$ or survival. ${ }^{* *} P<0.01,{ }^{* * *} P<0.001$. Asterisks in A, B, and $\mathbf{F}$ indicate treatment with ALT-803.

animals, received ALT-803 prior to MCMV infection with 2DG treatment (Figure 6A and Supplemental Figure $5 \mathrm{~A}$ ). NK depletion completely abrogated $m 157$-transgenic target cell clearance and significantly increased MCMV copy number at day 2 after infection (Figure 6, B and C). Target cell clearance and MCMV copy number were not affected by CD8 depletion (Supplemental Figure 5, B and C), suggesting that bystander activation of $\mathrm{CD} 8^{+}$memory $\mathrm{T}$ cells by ALT- 803 did not affect control of acute MCMV infection in naive mice. NK-depleted mice had significantly increased mortality (Figure 6D), while CD8 ${ }^{+} \mathrm{T}$ cell depletion had no effect on survival (Supplemental Figure 5D), demonstrating that the survival of ALT803 -treated mice is dependent on NK cells. NK depletion did not result in the $80 \%-100 \%$ lethality of $2 \mathrm{DG}$ treatment alone, indicating that ALT-803 may have some antiviral effects on NK1.1- cells (e.g., intraepithelial lymphocytes in the gut that can respond to IL-15, or DCs that produce and display IL-15; ref. 58). It is 
A

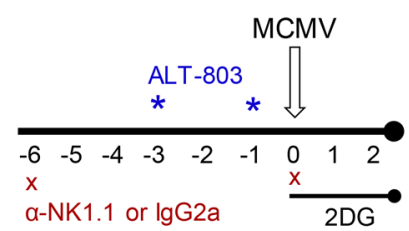

B

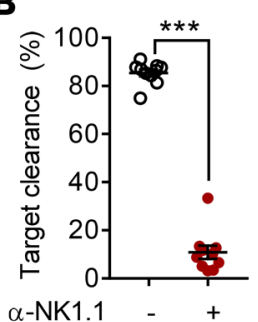

C

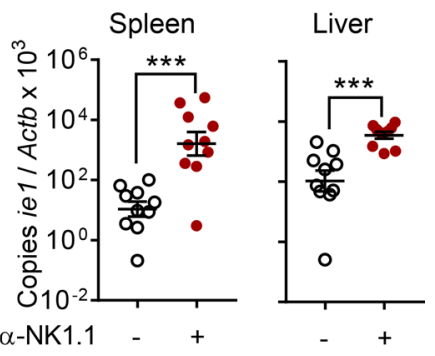

D

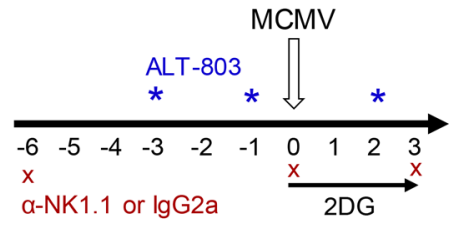

E

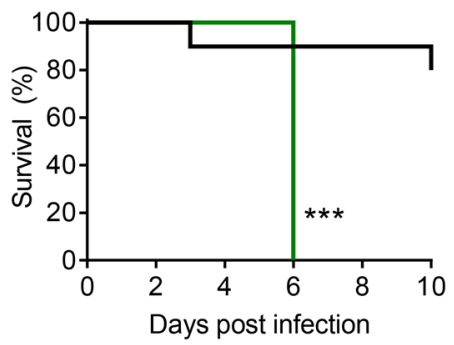

Vehicle Rapamycin

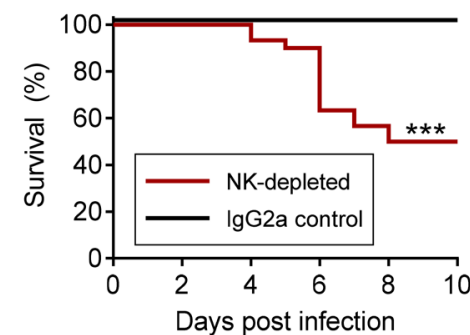

F

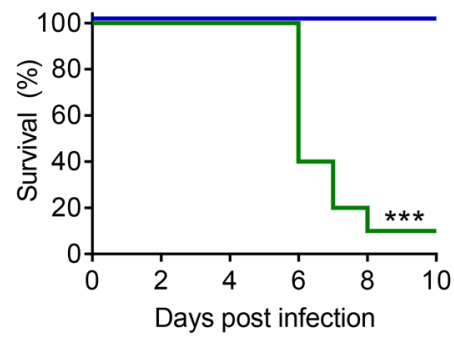

ALT-803 + Rapamycin PBS + Rapamycin
Figure 6. ALT-803 rescue of 2DG-treated mice requires NK cells, and ALT-803 priming can eliminate susceptibility to MCMV caused by $\mathrm{mTOR}$ inhibition. (A) Mice were either depleted of NK cells using $\alpha$-NK1.1 or treated with IgG2a; they were given 2 doses of ALT803 and infected with $1 \times 10^{5}$ PFU murine cytomegalovirus (MCMV). 2-Deoxy-D-glucose (2DC) was given every 24 hours, and mice were euthanized on day 2 for assessment of (B) clearance of m157-transgenic targets from the spleen and (C) viral copy number from the spleen and liver; they were measured by quantitative PCR and log-transformed $(n=10$ individuals/group, 2 separate experiments, 2-tailed $t$ test). (D) To assess susceptibility, an additional NK depletion was given at day 3 (red, $n=30$ ); control mice were treated with IgG2a (black, $n=23$ ). Mice were followed for 10 days (3 separate experiments). (E) Mice were infected with MCMV, given daily i.p. injections of $1.5 \mathrm{mg} / \mathrm{kg}$ rapamycin (green) or vehicle (black), and monitored for susceptibility ( $n=$ 10/group, 1 experiment). (F) ALT-803 was given to MCMV-infected, rapamycin-treated mice on the same schedule as for 2DG-treated mice, with doses at $-3,-1$, and +2 days relative to infection ( $n=10$ /group, 1 experiment). Data show mean \pm SEM or survival in $\mathbf{D}-\mathbf{F}$ analyzed by log-rank Mantel-Cox test. ${ }^{* *} P<0.001$. Asterisks in $\mathbf{A}$ and $\mathbf{D}$ indicate treatment with ALT-803.

also possible that NK cells remained or developed after antibody depletion treatment due to IL-15's strong survival and differentiation signaling; while there were no detectable NK cells in the blood or spleens of the majority of mice (data not shown), the efficacy of depletion in other organs was not evaluated.

mTOR inhibition leads to a similar survival defect with MCMV infection, which is rescued with IL-15 priming. High-dose IL-15 signaling induces mTOR signaling, and mTOR activity is linked to the upregulation of glycolysis $(11,59,60)$. We tested whether inhibition of mTORC1 led to susceptibility to MCMV infection, similar to 2DG treatment. Based on our finding that glycolytic inhibition impaired NK cell responses and led to mortality with MCMV, we hypothesized that mTOR signaling is required for NK cell-protective effects during MCMV, as was suggested by a prior study demonstrating increased viral copy number in rapamycin-treated MCMV-infected mice (59). Indeed, daily injection with $1.5 \mathrm{mg} / \mathrm{kg}$ rapamycin, an inhibitor of mTOR complex 1 (mTORC1), during MCMV infection led to susceptibility similar to 2DG treatment, with death 5-6 days after infection (Figure 6E). Also similar to 2DG, IL-15 priming with ALT-803 was protective and completely abrogated the effects of rapamycin during MCMV infection (Figure 6F). This suggests that, while IL-15 signaling has been described to upregulate mTOR and glycolysis, continued signaling through mTOR or maintenance of glycolysis is not required after IL-15 priming to sustain NK cell effector function.

Human NK cell cytotoxicity is reduced by treatment with $2 D G$. Relatively little is known about the effects of metabolism on human NK cell function, particularly cytotoxicity. To investigate whether human NK cells have a glycolytic requirement for cytotoxicity similar to the murine system, human NK cells were activated for 24 hours with IL-15 in the presence of varying doses of 2DG, then used in killing assays with K562 targets. Human NK cells were more resistant to 2DG than murine NK cells, but killing of K562 target cells was significantly decreased when cells were primed with IL-15 in the presence of 20-40 mM 2DG (Figure 7A). Actin accumulation and MTOC polarization were measured, and there were no significant defects in either (Figure 7, B and C). Thus, both human and 
A

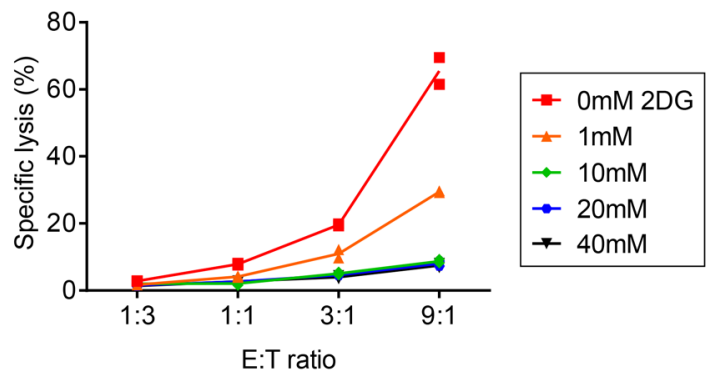

B

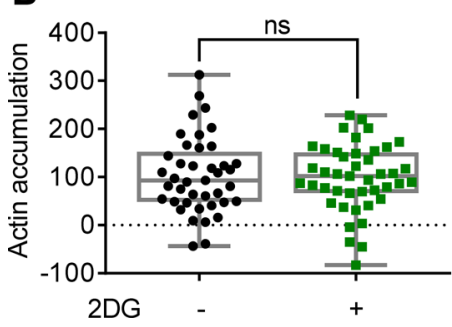

C

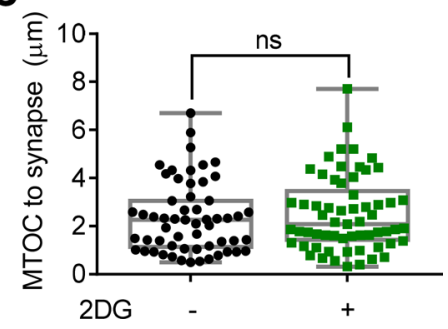

Figure 7. 2DG decreases human NK cell cytotoxicity. Human NK cells were isolated from healthy donors, enriched using RosetteSep, and cryopreserved. Cells were later thawed and activated in $100 \mathrm{ng} / \mathrm{ml}$ human IL-15 for 24 hours in various concentrations of 2DG. (A) NK cells were washed and cocultured with K562 targets for 4 hours at various E:T ratios; representative killing assay shown $(n=$ technical duplicate, representative of 6 donors across 4 experiments, mean with replicates shown). (B and C) NK cells from control conditions and 20-40 mM 2DG were cultured at a 2:1 ratio with $\mathrm{K} 562$ cells and imaged after 40 minutes. (B) Quantification of actin accumulation by area $x$ intensity of synapse - (NK + target) $(n=41-43$ conjugates/group, 1 experiment). (C) Quantification of distance from the microtubule organizing center (MTOC) to synapse in $\mu \mathrm{m}$ ( $n=67-61$ conjugates/group, 2 experiments). Gray boxes show median, 25th, and 75th percentiles. Neither $\mathbf{B}$ nor $\mathbf{C}$ was significant by 2-tailed $t$ test or Kolmogorov-Smirnov test. murine NK cell killing can be dampened by inhibition of glucose metabolism, though it is unclear if there are similar mechanisms regulating this effect.

Treatment of CMV reactivation in a patient with an $I L-15$ superagonist. Our results demonstrating that IL-15 treatment leads to enhanced protection against MCMV suggest that this cytokine may have therapeutic potential in patients. A 23-year-old CMV-seropositive male with dyskeratosis congenita and aplastic anemia received a matched sibling allogeneic HCT from his CMV-seronegative brother with full engraftment by 9 months (see Methods for case presentation). This patient experienced 5 episodes of CMV reactivation after transplant, which manifested as viremia without organ disease (Figure 8A). Three episodes of CMV viremia within the 9 months after HCT improved with antiviral therapy. A fourth episode at 18 months after HCT resolved with CMVpp65-specific $\mathrm{T}$ cell adoptive transfer.

At 51 months after HCT, the patient again experienced CMV reactivation. Because of recurrent CMV viremia, this patient was treated with compassionate-use ALT-803. Absolute numbers of peripheral blood NK cells were within normal limits at the time of ALT-803 administration. The patient received 4 weekly doses followed by 2 biweekly doses of ALT803 at $6 \mu \mathrm{g} / \mathrm{kg}$ per dose. No other antiviral therapy was given before or during the time of ALT-803 administration. Within 2 months, CMV viremia was below the limit of detection (Figure 8B), and CMV levels remained low for 7 weeks after ALT-803 therapy without additional antiviral therapy. Unfortunately, this patient experienced a recurrence of viremia when immunosuppressive therapy was required for treatment of posttransplant lymphoproliferative disorder.

NK cell function in this patient before treatment and 7 or 14 days after each dose of ALT-803 was evaluated. The patient had an elevated proportion of $\mathrm{CD} 57^{+} \mathrm{NKG} 2 \mathrm{C}^{+} \mathrm{NK}$ cells before treatment $(\sim 50 \%)$, consistent with the presence of CMV-adapted NK cells typically seen after CMV infection. This population of CMV-adapted NK cells was not altered by treatment with ALT-803 (data not shown). NK cell IFN- $\gamma$ production after overnight stimulation of cryopreserved peripheral blood mononuclear cells (PBMC) with IL-12 + IL-18 increased following ALT-803 treatment (Figure 8C). Although the absolute IFN- $\gamma$ response was lower in the patient's cells than in PBMC from healthy controls treated with IL-12 + IL-18 (mean \pm SD: $44.69 \% \pm 2.96 \%$ IFN- $\gamma$-producing NK cells, $n=8$ controls), the increased responsiveness in this patient suggests that NK cell function was augmented by ALT-803 therapy.

\section{Discussion}

Here, we examine the role of glycolysis in NK cell functions in vitro and in vivo. We found that the glucose metabolism inhibitor 2DG prevented IL-15-induced NK cell proliferation and priming of cytotoxicity in vitro. Using MCMV as an NK-dependent infection model, we demonstrate that administration of 2DG in vivo impaired NK-mediated target clearance and significantly increased susceptibility of mice to infection. These defects could be rescued in an NK-dependent manner with the IL-15 superagonist ALT-803. Treatment with an mTORC1 inhibitor led to a similar susceptibility to infection, which was also rescued by ALT-803 priming, suggested that IL-15-primed NK cells have mTOR- and glycolysis-independent sustained effector function. In a patient, treatment with ALT-803 also boosted NK cell function and was associated with clearance of CMV viremia, suggesting that targeting NK cell function in humans can lead to enhanced CMV control.

Our in vitro findings show that culture in 2DG blocks the ability of IL-15 to activate NK cell functions including proliferation, Gzmb production, and acquisition of enhanced cytotoxic functions. Cytotoxic defects were target cell-line specific, suggesting that certain pathways of NK cell activation may be able to 
A

CMV reactivation events

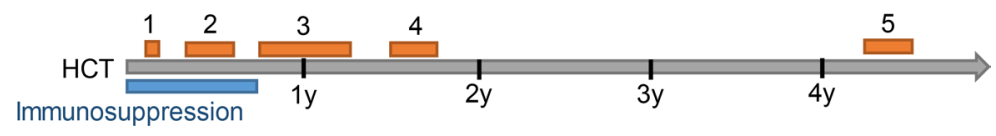

B
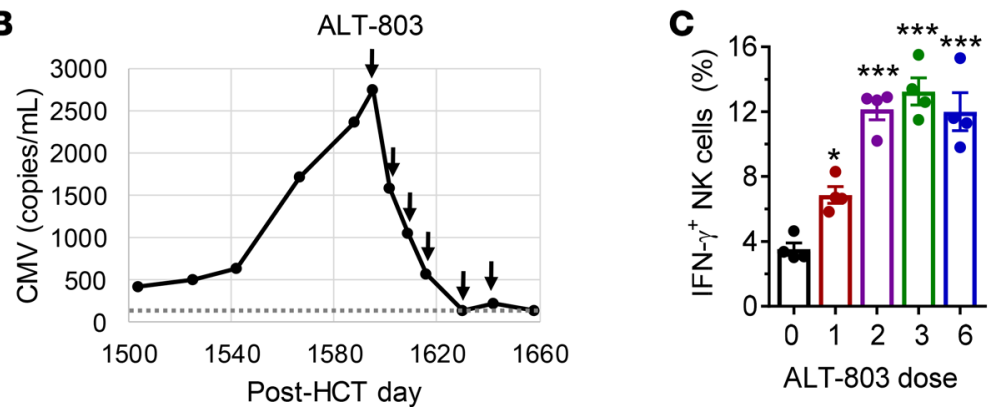

Figure 8. ALT-803 treatment of CMV reactivation in a posthematopoietic cell transplant patient. (A) The patient's course of cytomegalovirus (CMV) reactivation after hematopoietic cell transplant (HCT), with periods of viremia detectable by quantitative PCR assay shown as orange bars. Posttransplant immunosuppression is shown as a blue bar. Years 1-4 after HCT marked. (B) During reactivation event 5, CMV levels responded to treatment with ALT-803, as indicated by arrows. Points below limit of detection (dashed line) at 137 copies $/ \mathrm{ml}$ are rounded up. (C) To assess IFN- $\gamma$ production by the patient NK cells, peripheral blood mononuclear cells (PBMC) were collected and cryopreserved before ALT-803 treatment or 7-14 days after the listed ALT-803 dose. PBMC were thawed and stimulated with $10 \mathrm{ng} / \mathrm{ml} \mathrm{IL-12}$ and $100 \mathrm{ng} / \mathrm{ml} \mathrm{IL-18}$ for 16 hours; they were then washed and plated for an additional 5 hours ( $n=4$ technical replicates, 1 experiment, shown as mean \pm SEM, statistical significance in a 1-way ANOVA compared with the quantity of cells before dose 1 shown above relevant column). ${ }^{*} P<0.05$, ${ }^{* *} P<0.001$. overcome the dampened activation state of 2DG-treated NK cells or that certain pathways require glycolytic metabolites for signaling. Possible mechanisms for 2DG's effects on cytotoxicity include downregulation of Gzmb - leading to less cytotoxic granules - and altered actin accumulation at the IS. Actin accumulation is crucial for NK cell cytotoxicity, as evidenced by diseases associated with defects in actin accumulation such as Wiskott-Aldrich Syndrome, where NK cells fail to form conjugates or kill target cells (61). Although conjugation to target cells and MTOC polarization toward the synapse appeared normal in 2DG-treated NK cells, altered actin accumulation could be indicative of a less stable conjugation that could lead to slower or less effective killing, or could interfere with release of target cells and thus interfere with serial killing by NK cells. Interestingly, Wiskott-Aldrich NK cell defects can be reversed by IL-2 administration, as IL-2 activates an alternate actin polymerizing pathway $(62,63)$. Since IL-15 and IL-2 are closely related, this may provide a potential mechanism for IL-15 rescue of 2DG-induced deficits.

The observed target clearance defect caused by 2DG treatment resulted in higher viral replication, overwhelming infection, and mortality. MCMV infection has a steep $\mathrm{LD}_{50}$ curve (41), and loss of control of infection in 2DG-treated mice phenocopies mortality observed in mice depleted of $\mathrm{Ly} 49 \mathrm{H}^{+} \mathrm{NK}$ cells or total NK cells (24). Notably, not all NK cell functions were affected by $2 \mathrm{DG}$, with intact IFN- $\gamma$ production in vitro (10) and in vivo, and proliferation in vivo. Normal proliferation was somewhat surprising, as proliferating lymphocytes have been well described to rely on aerobic glycolysis $(64,65)$. This may be due to non-IL-15 signals in MCMV-infected mice, such as the presence of additional cytokines or activating receptor ligands, that could allow NK cells to proliferate despite glycolytic inhibition. The underlying mechanism for this glycolytic requirement for NK cell cytotoxicity can be partially explained by some of the findings here. 2DG decreases Gzmb upregulation in response to IL-2 + IL-12 (12) or IL-15 stimulation in vitro and after poly(I:C) injection in vivo (12). While 2DG did not appear to inhibit Gzmb upregulation in response to MCMV infection, this could be explained by different kinetics of Gzmb accumulation in response to different in vivo stimuli or by accumulation of Gzmb in 2DG-treated NK cells that are not actively degranulating and killing target cells. It is possible that killing defects in vivo are caused by changes in actin dynamics rather than Gzmb. Although actin accumulation defects did not alter conjugation in vitro, this may be due to the ready abundance and proximity of target cells. In vivo actin dynamics are crucial in NK cell trafficking, and minor defects in the ability to polymerize actin could lead to decreased numbers of NK cells at sites of infection.

Although 2DG administration is confounded by systemic effects, prior model systems have demonstrated that 2DG does not lead to generalized susceptibility to infection, as 2DG treatment did not increase susceptibility to listeria infection (66) and decreased virulence of other viruses, including HSV (67-69). Combined with our evidence that Ly49H-specific target clearance is decreased with 2DG and that deficient target clearance and survival can be rescued through IL-15, there is clear evidence of NK-specific defects with 2DG. However, NK cell-specific genetic KO of metabolic enzymes are necessary to further interrogate the role of metabolism in NK cell functions in vivo.

While NK cells require glycolysis during viral infection for optimal killing, pretreatment with IL-15 releases NK cells from their reliance on glucose metabolism, similar to what we previously observed in vitro for IFN- $\gamma$ (10). ALT-803 injection altered the metabolic rate of NK cells, leading to increased glycolysis and oxidative phosphorylation, and preserved NK cell-mediated target clearance in the presence 
of 2DG. Mice pretreated with ALT-803 were protected from MCMV infection when treated with either a glycolytic inhibitor or an inhibitor of mTORC1, suggesting that these pathways are not critical for IL-15primed NK cell antiviral responses.

The underlying mechanism for IL-15 reversing NK cell dependence on glucose metabolism and mTOR activation is unknown. IL-15 is well described to promote NK cell differentiation, expansion, Gzmb expression, cytokine production, and cytotoxicity $(32,70)$. We have previously shown that IL-15 upregulates NK cell metabolic capacity in vitro, especially glycolysis (10; here, we demonstrate that ALT-803 significantly increases metabolism in vivo, suggesting that IL-15 fundamentally alters cellular metabolic requirements. This altered metabolic state could lead to more flexible fuel usage by NK cells, allowing alternate fuels such as fatty acids, glutamine, or pyruvate to fuel NK cell function if glucose metabolism is limited. Alternately, we now have evidence that both cytokine-activated IFN- $\gamma$ production (10) and clearance of target cells during MCMV infection become less metabolically dependent with IL-15 pretreatment, suggesting that IL-15 increases the range of stimuli and functions that are metabolically independent. Recent studies have demonstrated a role for mTORC1 in IL-15 priming and NK cell responses to MCMV. mTORC1 activates glycolysis through HIF-1 and c-myc signaling, in addition to orchestrating broader cell growth programs (71). High-dose IL-15 signaling has been shown to be dependent on mTORC1 $(11,59)$, which likely contributes to IL-15-induced upregulation of glycolysis. However, here we demonstrate for the first time to our knowledge that, after IL-15 priming, mTORC1 signaling is not required for in vivo NK cell function, as shown by complete protection against an NK-dependent infection, MCMV, in rapamycin-treated mice pretreated with ALT-803. These findings distinguish the effects of mTORC1 signaling on priming versus maintenance of NK cell effector function.

The concept of augmenting the antiviral effects of NK cells with IL-15 agonists may be particularly relevant in patients after $\mathrm{HCT}$ and/or patients receiving immunosuppression targeting $\mathrm{T}$ cell responses. Following HCT, NK cells are the first lymphocyte population to emerge from the new BM and, thus, represent an important population to target for antileukemic and infection responses (72). In some cases, NK cells are reconstituted after HCT but are less functional for months, with reduced capacity for IFN- $\gamma$ production and cytotoxicity $(73,74)$. Here, we show that treatment with an IL-15 superagonist significantly boosted NK cell antiviral responses in mice and, in 1 patient, was associated with clearance of CMV infection in the absence of other antiviral therapy. Although limited conclusions can be drawn from a single patient, these studies suggest that clinical investigation of IL-15 agonists for treatment of refractory viral infections, particularly $\mathrm{CMV}$, is warranted. Here, we demonstrate that IL-15 activation of NK cells decreases NK cell reliance on glucose metabolism and mTOR signaling in vivo and may be a strategy to enhance NK cell function in metabolically challenging environments such as inflammatory and tumor microenvironments in patients.

In summary, our findings suggest that limiting glucose metabolism impairs NK cell killing and host antiviral responses to CMV, which can be rescued with IL-15-based priming. Such therapies may be particularly beneficial for patients after HCT, who may have normal numbers of NK cells but poor NK cell function. Additional investigations of the mechanisms by which metabolism alters NK function will be important for targeting these innate immune lymphocytes for immunotherapy and may provide insight into the metabolic regulation of killing in other cytotoxic lymphocytes.

\section{Methods}

Mice and MCMV infection. All mice were maintained in specific pathogen-free conditions, used between 6 and 12 weeks of age, and euthanized in accordance with institutional guidelines. C57BL/6 mice were purchased from Charles River Laboratories or from The Jackson Laboratory, and $m 157$-transgenic mice on the C57BL/ 6 background (42) were bred in-house. Seven- to 11-week-old C57BL/ 6 female mice were injected i.p. with $1 \times 10^{5} \mathrm{PFU}$ of Smith strain salivary gland MCMV (ATCC, prepared in BALB/c mice) or media as a control. 2DG (19 mg; MilliporeSigma) was given i.p. after infection and every 24 hours $(\sim 1 \mathrm{~g} / \mathrm{kg}$ per mouse) or PBS as a control. For proliferation assays, mice were injected with $2 \mathrm{mg}$ BrdU (BD Biosciences) i.p. 3 hours before harvest. For IL-15 superagonist treatment, $5 \mu \mathrm{g}$ ALT-803 (Altor BioScience) was injected i.p. 3 days and 1 day before MCMV infection and 2 days after infection. For NK and CD8 depletion, $100 \mu \mathrm{g}$ of $\alpha$-NK1.1 (PK136, BioXcell), $250 \mu \mathrm{g} \alpha$-CD8b (53-5.8, BioXcell), or $100 \mu \mathrm{g}$ murine IgG2a (C1.18.4, BioXcell) was administered i.p. 6 days before MCMV infection, the day of infection, and 3 days after infection. Mice were bled 24 hours before infection, or spleens were examined at the time of euthanasia to confirm $\mathrm{NK}$ or $\mathrm{CD}^{+} \mathrm{T}$ cell depletion by loss of $\mathrm{CD} 3^{-} \mathrm{NKp} 46^{+} \mathrm{DX} 5^{+}$or $\mathrm{CD}^{+} \mathrm{CD} 8 \mathrm{a}^{+}$ 
cells (data not shown). Rapamycin (Selleck Chemicals) was solubilized in 4\% EtOH (MilliporeSigma), 5\% Tween 80 (MilliporeSigma), 5\% PEG400 (MilliporeSigma), and water, and $30 \mu \mathrm{g}(\sim 1.5 \mathrm{mg} / \mathrm{kg}$ ) or an equivalent amount of vehicle was injected i.p. daily during MCMV infection.

In vitro assays. Primary splenocytes from female C57BL/6 mice were cultured in RPMI 1640 (Mediatech) supplemented with $10 \%$ FBS (MilliporeSigma), L-glutamine to a final concentration of $4 \mathrm{mM}$ (MilliporeSigma), $50 \mu \mathrm{M}$ 2-ME (MilliporeSigma), and antibiotics (penicillin/streptomycin). NK cells were enriched from spleens using negative-selection magnetic bead kits (Miltenyi Biotec and StemCell Technologies). Purity ranged from $55 \%-90 \%$ at time of plating, and 77\%-99\% after 72 hours of activation. For IL-15 activation, purified NK cells were cultured in $100 \mathrm{ng} / \mathrm{ml}$ IL-15 (PeproTech) for 72 hours with or without $1 \mathrm{mM} 2 \mathrm{DG}$. For proliferation assays, purified NK cells were stained with $1 \mu \mathrm{M}$ CFSE (Invitrogen) before culture.

For mouse NK cell killing assays, NK cells were washed and plated in V-bottom plates at estimated 10:1, 5:1, and 1:1 NK/target ratios with $1 \times 10^{4}$ CellTrace Violet-labeled target cells, including RMA-S (from W. Yokoyama, Washington University), Ba/F3-m157 (from W. Yokoyama; ref. 29), and YAC-1 (ATCC). Cell lines tested negative for mycoplasma. Effector/target ratios were calculated based on NK cell purity by flow cytometry. After 4 hours, cells were stained with 7-AAD (Calbiochem) for 15-30 minutes before flow cytometry, and specific lysis was calculated by subtracting the percentage of $7-\mathrm{AAD}^{+}$target cells from wells without effectors. For mouse NK cell conjugation assays, NK cells were labeled with CFSE and Ba/F3-m157 target cells with CellTrace Violet (CTV, Invitrogen). Cells were spun down at a 1:1 NK/ target ratio, incubated in $37^{\circ} \mathrm{C}$ for 15,30 , or 120 minutes, and then vortexed gently and fixed in $1 \%$ paraformaldehyde. Percent conjugated NK cells was calculated by calculating the percentage of $\mathrm{CFSE}^{+} \mathrm{CTV}^{+}$cells compared with all $\mathrm{CFSE}^{+}$cells.

Human NK cells from healthy donors were enriched using RosetteSep (StemCell). For killing assays, previously cryopreserved NK cells were plated in $100 \mathrm{ng} / \mathrm{ml}$ IL-15 (Miltenyi Biotec) and varying concentrations of 2DG for 22-26 hours, followed by incubation with labeled K562 targets for 4 hours at the indicated E:T ratios. Cells were then stained with 7-AAD, and specific lysis was calculated. For conjugation assays, NK cells were incubated with IL-15 and 2DG for 22-26 hours and then cocultured with K562 targets at a 2:1 ratio for 40-50 minutes. To assess patient NK cell function, cryopreserved PBMC from serial time points were thawed, incubated for 16 hours with human IL-12 (10 ng/ml) and IL-18 (100 ng/ml), washed, plated in replicates of 4 per sample, and incubated for an additional 5 hours prior to intracellular staining for IFN- $\gamma$ protein.

Flow cytometry. Cell viability was measured by live/dead stain (Zombie Yellow, BioLegend), followed by blocking with anti-FcyRIII (2.4G2, from W. Yokoyama) prior to surface staining. Cells were fixed with $1 \%$ paraformaldehyde for surface analysis, or fixed with Cytofix/Cytoperm (BD Bioscience) for intracellular staining. For intranuclear staining, cells were fixed in Cytofix/Cytoperm and Cytoperm Plus buffer (BD Biosciences) and DNAse treated. Data was acquired on a Cytek-modified FACScan (BD Biosciences and Cytek) or LSRFortessa (BD Biosciences); data was analyzed using FlowJo 7.6.5. Geometric MFI, and percent proliferated were calculated by FlowJo. The following fluorochrome-conjugated antibodies were used: CD3ع (145-2C11, BD Biosciences, BioLegend), CD4 (GK1.5, Leinco), CD8 $\alpha$ (53-6.7, BD Biosciences), CD11b (M1/70, BD Biosciences), CD11c (HL3, BD Biosciences), CD19 (eBio1D3, eBiosciences), IFN- $\gamma$ (XMG1.2, BioLegend), Ly49H (3D10, BioLegend), NK1.1 (PK136, BD Biosciences), NKp46 (29A1.4, BD Biosciences), Gzmb (GB12, Invitrogen), and BrdU (559319, BrdU kit, BD Biosciences). CD107a was stained by incubating splenocytes with $\alpha$-CD107a (1D4B, BD Biosciences) and GolgiStop (monensin, BD Biosciences) for 4 hours at $37^{\circ} \mathrm{C}$.

Confocal microscopy. Purified NK cells and Ba/F3-m157 or K562 targets were cocultured at a 2:1 ratio, incubated for 20 minutes at $37^{\circ} \mathrm{C}$, applied to poly-L-lysine-coated coverslips, and incubated for an additional 20 minutes. Slides were fixed in Cytofix/Cytoperm (BD Biosciences) $+0.1 \%$ Triton X-100 (MilliporeSigma) and then stained with the following antibodies: Ly49H (3D10, BioLegend), biotin-tubulin (236-10501, ThermoFisher), perforin (dG9, BioLegend), Gzmb (GB11, BioLegend), phalloidin (A12380, ThermoFisher), and streptavidin secondary antibody (S11222, ThermoFisher). Images were acquired on a Leica SP8 laser scanning confocal microscope equipped with a $100 \times 1.45$ NA objective. Excitation was performed by tunable white light laser, and emission was detected by $\mathrm{HyD}$ detector (Leica Biosystems). Data were acquired using Leica LASAF and analyzed in Volocity 6.1.1 (Perkin Elmer) and 6.3 (Quorum Technologies). Measurement of MTOC-synapse distance was single-blinded. Actin accumulation measurements were unblinded and consisted of the total area $\times$ average intensity of three $1-\mu \mathrm{m}$ squares spaced 
evenly or where there was sufficient actin staining. Actin accumulation was calculated by area $\times$ intensity of synapse - (NK + target) (75).

Extracellular flux. Extracellular flux assays were performed using a XF96 Analyzer (Seahorse Bioscience). Purified splenic NK cells were cultured for 72 hours in $100 \mathrm{ng} / \mathrm{ml}$ IL-15 with or without $1 \mathrm{mM}$ 2DG, or NK cells were purified from ALT-803- or control-treated mice. Cells were then washed and plated in at least duplicate for extracellular flux analysis of OCR and ECAR as previously described (10, 76). Glycolysis stress tests were performed in glucose-free DMEM (MilliporeSigma) supplemented with L-glutamine, sodium pyruvate, and 5\% dialyzed FBS. Drug concentrations during the assay were as follows: glucose, 10 mM; oligomycin, $100 \mathrm{nM}$; 2DG, $100 \mathrm{mM}$.

MCMV quantitative PCR. Tissue pieces $\left(5 \mathrm{~mm}^{3}\right)$ were minced and placed in Gentra Puregene Cell Lysis Buffer (Qiagen), and DNA was extracted following the manufacturer's recommended protocol and diluted to $20-50 \mathrm{ng} / \mathrm{ml}$. MCMV immediate-early gene 1-specific (ie1-specific) primers and probe were used to determine viral copy number as described (47) and normalized to $\beta$-actin (Actb). Reactions were run in duplicate on a 7500 Fast Real-Time PCR instrument (Applied Biosystems) with an absolute standard curve. The limit of reliable detection using the standard was 100 copies of MCMV, and all values below 100 were rounded up. Graphs represent ([copies MCMV ie1]/[copies Actb]) × 1,000. Statistics were performed on log-transformed data.

In vivo target clearance assay. Splenocytes were harvested from C57BL/6 mice and C57BL/6 m157-transgenic mice, stained with $10 \mu \mathrm{M}$ or $1 \mu \mathrm{M}$ CFSE, and mixed in a $\sim 1: 1$ ratio. Cells $\left(7 \times 10^{6}\right.$ to $\left.20 \times 10^{6}\right)$ were injected by tail vein into mice that had been infected with MCMV 2 days prior and treated daily with 2DG or PBS. Mice were treated with 2DG, but not ALT-803, prior to the clearance assay. The remaining labeled cells in the spleen were analyzed 4 hours after transfer. Percent target clearance was calculated by comparing the ratio of $m 157$-transgenic splenocytes (m157tg, targets) to WT splenocytes (nontargets) recovered to that originally injected: $1-(\% \mathrm{~m} 157 \mathrm{tg} / \% \mathrm{WT}$ cells at $4 \mathrm{hr}) /(\% \mathrm{~m} 157 \mathrm{tg} / \% \mathrm{WT}$ cells input $) \times 100$.

Patient case presentation. A 23-year-old CMV-seropositive male with dyskeratosis congenita and severe aplastic anemia received a matched sibling allogeneic HCT from his CMV-seronegative brother. Conditioning was reduced intensity (fludarabine $40 \mathrm{mg} / \mathrm{m}^{2} /$ day $\times 4$, cyclophosphamide $50 \mathrm{mg} / \mathrm{kg} \times 1$, total body irradiation $2 \mathrm{~Gy}$, and alemtuzumab $0.2 \mathrm{mg} / \mathrm{kg} / \mathrm{d} \times 5)$. Graft source was peripheral blood $\left(9.3 \times 10^{6} \mathrm{CD} 34^{+}\right.$ cells $/ \mathrm{kg}$ ), and the donor was his healthy brother (CMV seronegative). Graft-versus-host disease (GVHD) prophylaxis was with cyclosporine and mycophenolate mofetil, and viral prophylaxis was with high-dose acyclovir (800 mg 5 times daily) for approximately 25 days. Engraftment was complete as documented on day 28, and immunosuppressive medications for GVHD prophylaxis were discontinued 9 months after transplant. The patient developed no acute or chronic GVHD, relapse, or any major bacterial or fungal infection. However, the course of transplant was complicated by recurrent CMV reactivation without organ disease. Within the first 100 days after HCT, he had 1 CMV reactivation episode treated successfully with valganciclovir (Hoffman-La Roche), and the viremia resolved after 21 days. The second episode occurred 4 months after HCT and resolved after 3 months of treatment with valganciclovir followed by foscarnet. The third episode occurred 9 months after HCT and resolved after 6 months of treatment using maribavir (ClinicalTrials.gov, NCT01611974) followed by valganciclovir. The fourth episode occurred 18 months after HCT and resolved after 3 months with treatment using CMVpp65-specific T cell adoptive transfer ( 3 doses; ClinicalTrials.gov, NCT02136797; sponsored by Atara Biotherapeutics). This viral remission was durable and lasted for 31 months, at which time (51 months after HCT) the patient experienced another episode of reactivation. Mutation testing for antiviral resistance was negative.

Statistics. Statistics calculated in Prism 7 include mean, \pm SEM, median, 25th/75th percentiles, 2-tailed student's $t$ test, Log-rank Mantel-Cox test, ordinary 1-way ANOVA, or 2-way ANOVA. All treatment groups used in an experiment were included in the ANOVA analysis, including controls; in nonsurvival experiments, mice that appeared uninfected were excluded from analysis. Tukey's test and Sidak's tests were used to correct for multiple comparisons, and adjusted $P$ values are shown. The Kolmogorov-Smirnov test was calculated in Statistical Analysis System (SAS Institute) v9.4. $P$ values represent 2-tailed probability; $\alpha=0.05$. Data are presented with data averaged from each mouse or experiment as a separate point, and/or as mean \pm SEM.

Study approval. All animal studies were performed at Washington University or Baylor College of Medicine using an Animal Studies Committee (ASC) approved protocol, and they were conducted in accordance with ASC regulations. 
For human studies, written informed consent was obtained from the patient presented here in agreement with the guidelines of the University of Minnesota Human Research Protection Program. FDA approval for compassionate use of ALT-803 was obtained (Investigational New Drug [IND] application no. 132197).

\section{Author contributions}

AYM designed experiments, performed experiments, analyzed data, and wrote the manuscript. AR provided input for experimental design, collected clinical information, analyzed data, and edited the manuscript. MPK designed experiments and performed experiments. NS designed experiments and performed experiments. EKM designed experiments and performed experiments. JBA analyzed data. SKT provided a critical reagent and provided input for experimental design. SKA provided a critical reagent and experimental assistance. EKJ provided a critical reagent, provided input for experimental design, and edited the manuscript. HCW provided a critical reagent, provided input for experimental design, and edited the manuscript. JSM designed experiments, performed experiments, analyzed data, and edited the manuscript. TAF provided a critical reagent, provided input for experimental design, and edited the manuscript. EMM provided critical reagents, designed experiments, analyzed data, and edited the manuscript. ARF designed experiments, analyzed data, and edited the manuscript. MAC designed experiments, analyzed data, and wrote the manuscript.

\section{Acknowledgments}

The authors thank B. Parikh, C. Luke, and G. Silverman for experimental advice; H. Gu and M. Wallendorf for statistical analysis; and T. Vogel and A. Som for helpful scientific discussion. Work in the Cooper laboratory was supported by the NIH/NIAID (1K08AI085030 and R01AI127752), the Rheumatology Research Foundation, the Children's Discovery Institute and St. Louis Children's Hospital, and the American Association of Immunologists. AYM was supported by NIH training grant T32 GM07200 and NIAID fellowship 1F30AI129110. TAF was supported by R01AI102924. ARF was supported by R01AI078994.

Address correspondence to: Megan A. Cooper, 660 S. Euclid Avenue, Box 8208, Washington University School of Medicine, St. Louis, Missouri, 63110, USA. Phone: 314.286.0262; Email: Cooper_m@wustl.edu.

1. Fox CJ, Hammerman PS, Thompson CB. Fuel feeds function: energy metabolism and the T-cell response. Nat Rev Immunol. 2005;5(11):844-852.

2. Buck MD, O'Sullivan D, Pearce EL. T cell metabolism drives immunity. J Exp Med. 2015;212(9):1345-1360.

3. Galván-Peña S, O’Neill LA. Metabolic reprograming in macrophage polarization. Front Immunol. 2014;5:420.

4. Pearce EJ, Everts B. Dendritic cell metabolism. Nat Rev Immunol. 2015;15(1):18-29.

5. Lunt SY, Vander Heiden MG. Aerobic glycolysis: meeting the metabolic requirements of cell proliferation. Annu Rev Cell Dev Biol. 2011;27:441-464.

6. Jha AK, et al. Network integration of parallel metabolic and transcriptional data reveals metabolic modules that regulate macrophage polarization. Immunity. 2015;42(3):419-430.

7. Everts B, et al. TLR-driven early glycolytic reprogramming via the kinases TBK1-IKK $\varepsilon$ supports the anabolic demands of dendritic cell activation. Nat Immunol. 2014;15(4):323-332.

8. Chang CH, et al. Posttranscriptional control of T cell effector function by aerobic glycolysis. Cell. 2013;153(6):1239-1251.

9. De Rosa V, et al. Glycolysis controls the induction of human regulatory T cells by modulating the expression of FOXP3 exon 2 splicing variants. Nat Immunol. 2015;16(11):1174-1184.

10. Keppel MP, Saucier N, Mah AY, Vogel TP, Cooper MA. Activation-specific metabolic requirements for NK Cell IFN- $\gamma$ production. J Immunol. 2015;194(4):1954-1962.

11. Marçais A, et al. The metabolic checkpoint kinase mTOR is essential for IL-15 signaling during the development and activation of NK cells. Nat Immunol. 2014;15(8):749-757.

12. Donnelly RP, et al. mTORC1-dependent metabolic reprogramming is a prerequisite for NK cell effector function. J Immunol. 2014;193(9):4477-4484.

13. Keating SE, et al. Metabolic Reprogramming Supports IFN- $\gamma$ Production by CD56bright NK Cells. J Immunol. 2016;196(6):2552-2560.

14. Mah AY, Cooper MA. Metabolic Regulation of Natural Killer Cell IFN- $\gamma$ Production. Crit Rev Immunol. 2016;36(2):131-147.

15. Rölle A, Brodin P. Immune Adaptation to Environmental Influence: The Case of NK Cells and HCMV. Trends Immunol. 2016;37(3):233-243.

16. López-Botet M, Muntasell A, Vilches C. The CD94/NKG2C+ NK-cell subset on the edge of innate and adaptive immunity to human cytomegalovirus infection. Semin Immunol. 2014;26(2):145-151.

17. Wu Z, et al. Human cytomegalovirus-induced NKG2C(hi) CD57(hi) natural killer cells are effectors dependent on humoral antiviral immunity. J Virol. 2013;87(13):7717-7725 
18. Zhang T, Scott JM, Hwang I, Kim S. Cutting edge: antibody-dependent memory-like NK cells distinguished by FcR $\gamma$ deficiency. J Immunol. 2013;190(4):1402-1406.

19. Costa-Garcia M, Vera A, Moraru M, Vilches C, López-Botet M, Muntasell A. Antibody-mediated response of NKG2Cbright NK cells against human cytomegalovirus. J Immunol. 2015;194(6):2715-2724.

20. Lee J, et al. Epigenetic modification and antibody-dependent expansion of memory-like NK cells in human cytomegalovirus-infected individuals. Immunity. 2015;42(3):431-442.

21. Kuijpers TW, Baars PA, Dantin C, van den Burg M, van Lier RA, Roosnek E. Human NK cells can control CMV infection in the absence of T cells. Blood. 2008;112(3):914-915.

22. Bukowski JF, Woda BA, Habu S, Okumura K, Welsh RM. Natural killer cell depletion enhances virus synthesis and virus-induced hepatitis in vivo. J Immunol. 1983;131(3):1531-1538.

23. Scalzo AA, et al. The effect of the Cmv-1 resistance gene, which is linked to the natural killer cell gene complex, is mediated by natural killer cells. J Immunol. 1992;149(2):581-589.

24. Brown MG, et al. Vital involvement of a natural killer cell activation receptor in resistance to viral infection. Science 2001;292(5518):934-937.

25. Daniels KA, Devora G, Lai WC, O'Donnell CL, Bennett M, Welsh RM. Murine cytomegalovirus is regulated by a discrete subset of natural killer cells reactive with monoclonal antibody to Ly49H. J Exp Med. 2001;194(1):29-44.

26. Lee SH, et al. Susceptibility to mouse cytomegalovirus is associated with deletion of an activating natural killer cell receptor of the C-type lectin superfamily. Nat Genet. 2001;28(1):42-45.

27. Orange JS, Biron CA. Characterization of early IL-12, IFN-alphabeta, and TNF effects on antiviral state and NK cell responses during murine cytomegalovirus infection. J Immunol. 1996;156(12):4746-4756.

28. Dokun AO, Kim S, Smith HR, Kang HS, Chu DT, Yokoyama WM. Specific and nonspecific NK cell activation during virus infection. Nat Immunol. 2001;2(10):951-956.

29. Smith HR, et al. Recognition of a virus-encoded ligand by a natural killer cell activation receptor. Proc Natl Acad Sci USA. 2002;99(13):8826-8831.

30. Arase H, Mocarski ES, Campbell AE, Hill AB, Lanier LL. Direct recognition of cytomegalovirus by activating and inhibitory NK cell receptors. Science. 2002;296(5571):1323-1326.

31. French AR, et al. DAP12 signaling directly augments proproliferative cytokine stimulation of NK cells during viral infections J Immunol. 2006;177(8):4981-4990.

32. Fehniger TA, et al. Acquisition of murine NK cell cytotoxicity requires the translation of a pre-existing pool of granzyme B and perforin mRNAs. Immunity. 2007;26(6):798-811.

33. Orr MT, et al. Ly49H signaling through DAP10 is essential for optimal natural killer cell responses to mouse cytomegalovirus infection. J Exp Med. 2009;206(4):807-817.

34. Berry R, et al. Targeting of a natural killer cell receptor family by a viral immunoevasin. Nat Immunol. 2013;14(7):699-705.

35. Ljunggren HG, Kärre K. Host resistance directed selectively against H-2-deficient lymphoma variants. Analysis of the mechanism. J Exp Med. 1985;162(6):1745-1759.

36. Kärre K, Ljunggren HG, Piontek G, Kiessling R. Selective rejection of H-2-deficient lymphoma variants suggests alternative immune defence strategy. Nature. 1986;319(6055):675-678.

37. Diefenbach A, Jamieson AM, Liu SD, Shastri N, Raulet DH. Ligands for the murine NKG2D receptor: expression by tumor cells and activation of NK cells and macrophages. Nat Immunol. 2000;1(2):119-126.

38. Jamieson AM, Diefenbach A, McMahon CW, Xiong N, Carlyle JR, Raulet DH. The role of the NKG2D immunoreceptor in immune cell activation and natural killing. Immunity. 2002;17(1):19-29.

39. Guerra N, et al. NKG2D-deficient mice are defective in tumor surveillance in models of spontaneous malignancy. Immunity. 2008;28(4):571-580

40. Mace EM, et al. Cell biological steps and checkpoints in accessing NK cell cytotoxicity. Immunol Cell Biol. 2014;92(3):245-255

41. Geurs TL, Hill EB, Lippold DM, French AR. Sex differences in murine susceptibility to systemic viral infections. $J$ Autoimmun. 2012;38(2-3):J245-J253.

42. Tripathy SK, et al. Continuous engagement of a self-specific activation receptor induces NK cell tolerance. $J$ Exp Med 2008;205(8):1829-1841

43. Dalod M, et al. Interferon alpha/beta and interleukin 12 responses to viral infections: pathways regulating dendritic cell cytokine expression in vivo. J Exp Med. 2002;195(4):517-528.

44. Tay CH, Welsh RM. Distinct organ-dependent mechanisms for the control of murine cytomegalovirus infection by natural killer cells. J Virol. 1997;71(1):267-275.

45. Loh J, Chu DT, O'Guin AK, Yokoyama WM, Virgin HW. Natural killer cells utilize both perforin and gamma interferon to regulate murine cytomegalovirus infection in the spleen and liver. $J$ Virol. 2005;79(1):661-667.

46. Fodil N, et al. Specific dysregulation of IFN $\gamma$ production by natural killer cells confers susceptibility to viral infection. PLoS Pathog. 2014;10(12):e1004511.

47. Parikh BA, Piersma SJ, Pak-Wittel MA, Yang L, Schreiber RD, Yokoyama WM. Dual Requirement of Cytokine and Activation Receptor Triggering for Cytotoxic Control of Murine Cytomegalovirus by NK Cells. PLoS Pathog. 2015;11(12):e1005323.

48. Xu W, et al. Efficacy and mechanism-of-action of a novel superagonist interleukin-15: interleukin-15 receptor $\alpha \mathrm{Su} / \mathrm{Fc}$ fusion complex in syngeneic murine models of multiple myeloma. Cancer Res. 2013;73(10):3075-3086.

49. Gomes-Giacoia E, et al. Intravesical ALT-803 and BCG treatment reduces tumor burden in a carcinogen induced bladder can cer rat model; a role for cytokine production and NK cell expansion. PLoS One. 2014;9(6):e96705.

50. Rosario M, et al. The IL-15-Based ALT-803 Complex Enhances Fc $\gamma$ RIIIa-Triggered NK Cell Responses and In Vivo Clearance of B Cell Lymphomas. Clin Cancer Res. 2016;22(3):596-608.

51. Kim PS, et al. IL-15 superagonist/IL-15R $\alpha$ Sushi-Fc fusion complex (IL-15SA/IL-15R $\alpha$ Su-Fc; ALT-803) markedly enhances specific subpopulations of $\mathrm{NK}$ and memory CD8+ T cells, and mediates potent anti-tumor activity against murine breast and colon carcinomas. Oncotarget. 2016;7(13):16130-16145.

52. Mathios D, et al. Therapeutic administration of IL-15 superagonist complex ALT-803 leads to long-term survival and durable 
antitumor immune response in a murine glioblastoma model. Int J Cancer. 2016;138(1):187-194.

53. Basher F, Jeng EK, Wong H, Wu J. Cooperative therapeutic anti-tumor effect of IL-15 agonist ALT-803 and co-targeting soluble NKG2D ligand sMIC. Oncotarget. 2016;7(1):814-830.

54. Allegrezza MJ, et al. IL15 Agonists Overcome the Immunosuppressive Effects of MEK Inhibitors. Cancer Res. 2016;76(9):2561-2572.

55. Felices M, et al. IL-15 super-agonist (ALT-803) enhances natural killer (NK) cell function against ovarian cancer. Gynecol Oncol. 2017;145(3):453-461.

56. Seay K, et al. In Vivo Activation of Human NK Cells by Treatment with an Interleukin-15 Superagonist Potently Inhibits Acute In Vivo HIV-1 Infection in Humanized Mice. J Virol. 2015;89(12):6264-6274.

57. Jones RB, et al. A Subset of Latency-Reversing Agents Expose HIV-Infected Resting CD4+ T-Cells to Recognition by Cytotoxic T-Lymphocytes. PLoS Pathog. 2016;12(4):e1005545.

58. Schluns KS, Nowak EC, Cabrera-Hernandez A, Puddington L, Lefrançois L, Aguila HL. Distinct cell types control lymphoid subset development by means of IL-15 and IL-15 receptor alpha expression. Proc Natl Acad Sci USA. 2004;101(15):5616-5621.

59. Nandagopal N, Ali AK, Komal AK, Lee SH. The Critical Role of IL-15-PI3K-mTOR Pathway in Natural Killer Cell Effector Functions. Front Immunol. 2014;5:187.

60. Düvel K, et al. Activation of a metabolic gene regulatory network downstream of mTOR complex 1. Mol Cell. 2010;39(2):171-183

61. Orange JS, et al. Wiskott-Aldrich syndrome protein is required for NK cell cytotoxicity and colocalizes with actin to NK cell-activating immunologic synapses. Proc Natl Acad Sci USA. 2002;99(17):11351-11356.

62. Gismondi A, et al. Impaired natural and CD16-mediated NK cell cytotoxicity in patients with WAS and XLT: ability of IL-2 to correct NK cell functional defect. Blood. 2004;104(2):436-443.

63. Orange JS, et al. IL-2 induces a WAVE2-dependent pathway for actin reorganization that enables WASp-independent human NK cell function. J Clin Invest. 2011;121(4):1535-1548.

64. Vander Heiden MG, Cantley LC, Thompson CB. Understanding the Warburg effect: the metabolic requirements of cell proliferation. Science. 2009;324(5930):1029-1033.

65. Warburg O, Gawehn K, Geissler AW. [Metabolism of leukocytes]. Z Naturforsch B. 1958;13B(8):515-516.

66. Miller ES, Bates RA, Koebel DA, Fuchs BB, Sonnenfeld G. 2-deoxy-D-glucose-induced metabolic stress enhances resistance to Listeria monocytogenes infection in mice. Physiol Behav. 1998;65(3):535-543.

67. Kilbourne ED. Inhibition of influenza virus multiplication with a glucose antimetabolite (2-deoxy-D-glucose). Nature. 1959;183(4656):271-272.

68. Schnitzer TJ, Hodes DS, Gerin J, Camargo E, Chanock RM. Effect of 2-deoxy-D-glucose and glucosamine on the growth and functions of respiratory syncytial and parainfluenza 3 viruses. Virology. 1975;67(1):306-309.

69. Courtney RJ, Steiner SM, Benyesh-Melnick M. Effects of 2-deoxy-D-glucose on herpes simplex virus replication. Virology. 1973;52(2):447-455.

70. Marçais A, Viel S, Grau M, Henry T, Marvel J, Walzer T. Regulation of mouse NK cell development and function by cytokines. Front Immunol. 2013;4:450.

71. Weichhart T, Hengstschläger M, Linke M. Regulation of innate immune cell function by mTOR. Nat Rev Immunol. 2015;15(10):599-614.

72. Ullah MA, Hill GR, Tey SK. Functional Reconstitution of Natural Killer Cells in Allogeneic Hematopoietic Stem Cell Transplantation. Front Immunol. 2016;7:144.

73. Foley B, et al. NK cell education after allogeneic transplantation: dissociation between recovery of cytokine-producing and cytotoxic functions. Blood. 2011;118(10):2784-2792.

74. Pical-Izard C, et al. Reconstitution of natural killer cells in HLA-matched HSCT after reduced-intensity conditioning: impact on clinical outcome. Biol Blood Marrow Transplant. 2015;21(3):429-439.

75. Banerjee PP, Orange JS. Quantitative measurement of F-actin accumulation at the NK cell immunological synapse. J Immunol Methods. 2010;355(1-2):1-13.

76. Keppel MP, Cooper MA. Assessment of NK Cell Metabolism. Methods Mol Biol. 2016;1441:27-42. 